\title{
Accretive and Sectorial Extensions of Nonnegative Symmetric Operators
}

\author{
Yury Arlinskiı̌ • Yury Kovalev • \\ Eduard Tsekanovskiı̌
}

Received: 23 December 2010 / Accepted: 24 June 2011 / Published online: 19 August 2011

(C) The Author(s) 2011. This article is published with open access at Springerlink.com

\begin{abstract}
We present the solution to the Phillips-Kato restricted extension problem about description and parametrization of the domains of all maximal accretive and sectorial quasi-self-adjoint extensions $\widetilde{S}\left(S \subset \widetilde{S} \subset S^{*}\right)$ of a closed, densely defined nonnegative operator $S$ in some Hilbert space. This description and parametrization are presented in terms of some sort of an analogy of von Neumann's formulas for quasiself-adjoint extensions. We use the approach proposed by Arlinskiı̌ and Tsekanovskiŭ (Integr Equ Oper Theory 51:319-356, 2005) and our new formulas match the corresponding ones in the case of nonnegative self-adjoint extensions of $S$. An application to operators corresponding to finite number $\delta^{\prime}$-interactions on the real line is given as well as to the parametrization of all resolvents of maximal accretive extensions.
\end{abstract}

Keywords Symmetric operator - Quasi-self-adjoint extensions · Friedrichs extension $\cdot$ KreĬn-von Neumann extension $\cdot m$-accretive operator $\cdot m$-sectorial operator

Dedicated to Franek Szafraniec on the occasion of his 70th birthday anniversary.

Communicated by Guest Editors L. Littlejohn and J. Stochel.

Y. Arlinskiı̆ $(\varangle) \cdot$ Y. Kovalev

Department of Mathematical Analysis, East Ukrainian National University, Kvartal Molodyozhny 20-A, Lugansk 91034, Ukraine

e-mail: yury.kovalev.lugansk@gmail.com

Y. Kovalev

e-mail: canadez@ukr.net

E. Tsekanovskiı̌

Department of Mathematics, Niagara University, P.O. Box 2044,

Niagara Falls, NY 14109, USA

e-mail: tsekanov@niagara.edu 


\section{Introduction}

Let $S$ be a closed densely defined symmetric operator acting in the Hilbert space $\mathfrak{H}$. An operator $\widetilde{S}$ is called a quasi-self-adjoint extension of $S$ if

$$
S \subset \widetilde{S} \subset S^{*} .
$$

Suppose $S$ is nonnegative, i.e., $(S f, f) \geq 0$ for all $f \in \operatorname{Dom}(S)$. We are interested in a solution of the restricted Phillips-Kato extension problem about description and parametrization of the domains of all quasi-self-adjoint maximal accretive (m-accretive) and maximal sectorial ( $\mathrm{m}$-sectorial) with vertex at zero [35] extensions $\widetilde{S}$ of $S$. This problem is a special case of the general Phillips problem $[45,46]$ on parametrization of all $\mathrm{m}$-accretive extensions for the given densely defined accretive operator. It was established by Phillips that any closed densely defined accretive operator admits an m-accretive extension. In order to obtain a description of all $\mathrm{m}$-accretive extension Phillips proposed to use the approach connected with geometry of spaces with indefinite inner product. His approach has been applied in [28,29] for $m$-accretive boundary value problems generated by positive definite ordinary differential expression, and in [44] for an abstract positive definite symmetric operator with finite defect numbers. The fractional-linear transformation reduces the Phillips problem to the dual problem of a parametrization of all contractive extensions for a given non-densely defined contraction. Such parametrization has been obtained in [21].

The problem of existence and description of all quasi-self-adjoint m-accretive extensions of a nonnegative symmetric operator via fractional-linear transformation has been solved in [14] and via abstract boundary conditions in $[4,25,26,36,43]$. We refer on this matter to the survey [18] where one can find information about various approaches to the extension problem of nonnegative symmetric operators. In this paper we give an intrinsic description and parametrization (in terms of some analogy of von Neumann's formulas for quasi-self-adjoint extensions) of the domains of all $\mathrm{m}$-accretive and m-sectorial quasi-self-adjoint extensions of nonnegative $S$. For this purpose we develop and apply the method recently proposed in [15-17] for the characterization of nonnegative self-adjoint extensions. Main results of this paper have been announced in [10].

We keep the following notations: $\mathbf{L}\left(\mathfrak{H}_{1}, \mathfrak{H}_{2}\right)$ denotes the Banach space of all continuous linear operators acting from the Hilbert space $\mathfrak{H}_{1}$ into the Hilbert space $\mathfrak{H}_{2}, \mathbf{L}(\mathfrak{H})=\mathbf{L}(\mathfrak{H}, \mathfrak{H})$ and $\operatorname{Dom}(T), \quad \operatorname{Ran}(T), \operatorname{Ker} T, \rho(T)$ denote the domain, the range, the null-space and the resolvent set of a linear operator $T$, respectively. The Moore-Penrose inverse of a a self-adjoint operator $B$ is defined by $\widehat{B}^{-1}$, i.e., by definition $\widehat{B}^{-1}=(B \backslash \operatorname{Ran}(B))^{-1} \oplus 0 \uparrow \operatorname{Ker} B$. Symbols $\mathbb{C}_{+}\left(\mathbb{C}_{-}\right)$and $\Pi_{+}\left(\Pi_{-}\right)$ denote the upper (lower) and right (left) open half-planes of the complex plane $\mathbb{C}$, respectively. 


\section{Preliminaries}

\subsection{Symmetric, Self-Adjoint, and Dissipative Operators}

Let $\mathfrak{H}$ be a separable Hilbert space with the inner product $(\cdot, \cdot)$. A closed linear operator $S$ in $\mathfrak{H}$ is called symmetric if its domain $\operatorname{Dom}(S)$ is a dense linear manifold in $\mathfrak{H}$ and the quadratic form $(S f, f)$ takes real values for all $f \in \operatorname{Dom}(S)$. This means that $(S f, g)=(f, S g)$ for all $f, g \in \operatorname{Dom}(S)$. Equivalently $S \subset S^{*}$, where $S^{*}$ is the adjoint operator to $S$. An operator $A$ is called self-adjoint if $A=A^{*}$. It is well known that $\rho(A) \supset \mathbb{C}_{+} \cup \mathbb{C}_{-}$.

An operator $T$ in $\mathfrak{H}$ is called dissipative (anti-dissipative) if

$$
\operatorname{Im}(T f, f) \geq 0 \quad(\operatorname{Im}(T f, f) \leq 0) \quad \text { for all } f \in \operatorname{Dom}(T) .
$$

A dissipative (anti-dissipative) operator $T$ is called maximal dissipative (maximal anti-dissipative) if $\rho(T) \cap \mathbb{C}_{-} \neq \emptyset\left(\rho(T) \cap \mathbb{C}_{+} \neq \emptyset\right)$.

\subsection{Nonnegative Symmetric, Accretive, and Sectorial Operators}

A symmetric operator $S$ is called nonnegative (we will write $S \geq 0$ ) if $(S f, f) \geq 0$ for all $f \in \operatorname{Dom}(S)$.

If $B$ and $C$ are two bounded self-adjoint operators acting on $\mathfrak{H}$, then the notation $B \geq C$ means that the operator $B-C \geq 0$. As is well known the square root $B^{1 / 2}$ of a nonnegative self-adjoint operator $B$ has the following properties:

$$
\begin{aligned}
\operatorname{Ran}\left(B^{1 / 2}\right) & =\left\{g \in \mathfrak{H}: \sup _{f \in \operatorname{Dom}(B)} \frac{|(f, g)|^{2}}{(B f, f)}<\infty\right\}, \\
\left\|\widehat{B}^{-1 / 2} g\right\|^{2} & =\sup _{f \in \mathfrak{H}} \frac{|(f, g)|^{2}}{(B f, f)}, \quad g \in \operatorname{Ran}\left(B^{1 / 2}\right), \\
\lim _{z \uparrow 0}\left((B-z I)^{-1} g, g\right) & = \begin{cases}\left\|\widehat{B}^{-1 / 2} g\right\|^{2}, & g \in \operatorname{Ran}\left(B^{1 / 2}\right), \\
+\infty, & g \in \mathfrak{H} \backslash \operatorname{Ran}\left(B^{1 / 2}\right),\end{cases}
\end{aligned}
$$

cf. [41].

A linear operator $T$ in $\mathfrak{H}$ is called accretive if $\operatorname{Re}(T f, f) \geq 0$ for all $f \in \operatorname{Dom}(T)$ and maximal accretive ( $m$-accretive) if it is accretive and has no accretive extensions in $\mathfrak{H}$. The following statements are equivalent [46]:

(i) the operator $T$ is $m$-accretive;

(ii) the operator $T$ is accretive and $\rho(T) \cap \Pi_{-} \neq \emptyset$;

(iii) the operators $T$ and $T^{*}$ are accretive.

The resolvent set $\rho(T)$ of $m$-accretive operator contains the open left half-plane $\Pi_{-}$and

$$
\left\|\left(T-z I_{\mathfrak{H}}\right)^{-1}\right\| \leq \frac{1}{|\operatorname{Re} z|}, \quad \operatorname{Re} z<0
$$


It is well known [35] that if $T$ is $m$-accretive operator, then the one-parameter semigroup

$$
T(t)=\exp (-t T), \quad t \geq 0
$$

is contractive. Conversely [35], if $\{T(t)\}_{t \geq 0}$ is a strongly continuous one-parameter contractive semigroup in a Hilbert space $\mathfrak{H}$, with $T(0)=I_{\mathfrak{H}}\left(C_{0}\right.$-semigroup), then the generator $T$ of $T(t)$ :

$$
T u:=\lim _{t \rightarrow+0} \frac{\left(I_{\mathfrak{H}}-T(t)\right) u}{t}, u \in \operatorname{Dom}(T),
$$

where the domain Dom $(T)$ is defined by the condition:

$$
\operatorname{Dom}(T)=\left\{u \in \mathfrak{H}: \lim _{t \rightarrow+0} \frac{\left(I_{\mathfrak{H}}-T(t)\right) u}{t} \text { exists }\right\},
$$

is an $m$-accretive operator in $\mathfrak{H}$.

Let $\alpha \in(0, \pi / 2)$ and denote by $\mathcal{S}(\alpha)$ the following sector of the complex plane:

$$
\mathcal{S}(\alpha)=\{z \in \mathbb{C}:|\arg z| \leq \alpha\} .
$$

A linear operator $T$ in a Hilbert space $\mathfrak{H}$ is said to be sectorial with vertex at the origin and semi-angle $\alpha$, if its numerical range

$$
W(T)=\{(T f, f):\|f\|=1, f \in \operatorname{Dom}(T)\}
$$

is contained in the sector $\mathcal{S}(\alpha)$, cf. [35]. This condition is equivalent to

$$
|\operatorname{Im}(T f, f)| \leq \tan \alpha \operatorname{Re}(T f, f) \text { for all } f \in \operatorname{Dom}(T) .
$$

If $T$ is $m$-accretive and sectorial, then $T$ is called maximal sectorial. A maximal sectorial operator $T$ is densely defined and its adjoint $T^{*}$ is also a maximal sectorial operator. In the sequel we will call such operators $\mathrm{m}-\alpha$-sectorial. Clearly, nonnegative (self-adjoint) operator is $\mathrm{m}$ - 0 -sectorial. The resolvent set of $\mathrm{m}$ - $\alpha$-sectorial operator $T$ contains the set $\mathbb{C} \backslash \mathcal{S}(\alpha)$ and

$$
\left\|\left(T-z I_{\mathfrak{H}}\right)^{-1}\right\| \leq \frac{1}{\operatorname{dist}(z, \mathcal{S}(\alpha))}, \quad z \in \mathbb{C} \backslash \mathcal{S}(\alpha) .
$$

It is well-known [35] that a $C_{0}$-semigroup $T(t)=\exp (-t T), t \geq 0$ has contractive and holomorphic continuation into the sector $\mathcal{S}(\pi / 2-\alpha)$ if and only if the generator $T$ is m- $\alpha$-sectorial operator.

\subsection{Classes $C_{\mathfrak{H}}(\alpha)$}

Let $\alpha \in(0, \pi / 2)$. A bounded operator $T$ on a Hilbert space $\mathfrak{H}$ is said to belong to the class $C_{\mathfrak{H}}(\alpha)$ [3] if

$$
\|T \sin \alpha \pm i \cos \alpha I\| \leq 1 .
$$


Clearly, $T$ belongs to $C_{\mathfrak{H}}(\alpha)$ if and only if $T^{*}$ belongs to $C_{\mathfrak{H}}(\alpha)$. Put

$$
D_{T}=\left(I-T^{*} T\right)^{1 / 2}, \mathfrak{D}_{T}=\overline{\operatorname{Ran}}\left(D_{T}\right) .
$$

Condition (2.3) is equivalent to each of the following two:

$$
\left|\left(T_{I} f, f\right)\right| \leq \frac{\tan \alpha}{2}\left\|D_{T} f\right\|^{2} \text { for all } f \in \mathfrak{H}
$$

or

$$
\text { the operator }\left(I-T^{*}\right)(I+T) \text { is } \mathrm{m} \text { - } \alpha \text {-sectorial. }
$$

cf. [5]. Moreover, it follows from (2.3) that the operators belonging to $C_{\mathfrak{H}}(\alpha)$ are contractive. From (2.4) and (2.3) it is naturally to consider all self-adjoint contractions and all contractions in $\mathfrak{H}$ as operators of the classes $C_{\mathfrak{H}}(0)$ and $C_{\mathfrak{H}}(\pi / 2)$, respectively.

Note that the linear fractional transformation $T=(I-S)(I+S)^{-1}$ of an $\mathrm{m}-\alpha-$ sectorial operator $S$ is an operator of the class $C_{\mathfrak{H}}(\alpha)$. Let

$$
\widetilde{C}_{\mathfrak{H}}=\bigcup\left\{C_{\mathfrak{H}}(\alpha): \alpha \in[0, \pi / 2)\right\}
$$

Some properties of the operators in the class $\widetilde{C}_{\mathfrak{H}}$ were studied in $[3,5]$. In particular, in [3] it was proved that $T \in \widetilde{C}_{\mathfrak{H}}$ implies that

$$
\operatorname{Ran}\left(D_{T^{n}}\right)=\operatorname{Ran}\left(D_{T^{* n}}\right)=\operatorname{Ran}\left(D_{T_{R}}\right), \quad n=1,2, \ldots,
$$

where $T_{R}$ is the real part of $T$. Furthermore it was proved in [3] that the subspace $\mathfrak{D}_{T}$ reduces the operator $T$, that the operator $T\left\lceil\operatorname{Ker} D_{T}\right.$ is self-adjoint and unitary, and that $T\left\lceil\mathfrak{D}_{T}\right.$ is a completely non-unitary contraction of the class $C_{00}$, i.e.,

$$
\lim _{n \rightarrow \infty} T^{n} f=\lim _{n \rightarrow \infty} T^{* n} f=0 \text { for all } f \in \mathfrak{D}_{T},
$$

cf. [48].

\subsection{Linear Relations}

As is well known a linear relation (1.r.) in a Hilbert space $\mathcal{H}$ is a subspace in $\mathcal{H}^{2}:=$ $\mathcal{H} \oplus \mathcal{H}$ equipped by the standard inner product

$$
(\vec{u}, \vec{v})_{\mathcal{H}^{2}}=\left(u_{1}, v_{1}\right)+\left(u_{2}, v_{2}\right)
$$

for $\vec{u}=\left\langle u_{1}, u_{2}\right\rangle, \vec{v}=\left\langle v_{1}, v_{2}\right\rangle \in \mathcal{H}^{2}$. In particular the graph

$$
\operatorname{Gr}(T)=\{\langle h, T h\rangle, h \in \operatorname{Dom}(T)\}
$$


of a linear operator $T$ in $\mathcal{H}$ provides an example of l.r. If $\mathbf{T}$ is a 1.r., then by definition

$$
\begin{aligned}
\operatorname{Dom}(\mathbf{T}) & =\left\{x \in \mathcal{H}:\left\langle x, x^{\prime}\right\rangle \in \mathbf{T} \text { for some } x^{\prime} \in \mathcal{H}\right\}, \\
\operatorname{Ran}(\mathbf{T}) & =\left\{x^{\prime} \in \mathcal{H}:\left\langle x, x^{\prime}\right\rangle \in \mathbf{T} \text { for some } x \in \mathcal{H}\right\}, \\
\operatorname{Ker} \mathbf{T} & =\{x \in \operatorname{Dom}(\mathbf{T}):\langle x, 0\rangle \in \mathbf{T}\}, \\
\lambda \mathbf{T} & =\left\{\left\langle x, \lambda x^{\prime}\right\rangle,\left\langle x, x^{\prime}\right\rangle \in \mathbf{T}\right\}, \\
\mathbf{T}^{-1} & =\left\{\left\langle x^{\prime}, x\right\rangle:\left\langle x, x^{\prime}\right\rangle \in \mathbf{T}\right\} .
\end{aligned}
$$

For $x \in \operatorname{Dom}(\mathbf{T})$ we set

$$
\mathbf{T} x=\left\{x^{\prime} \in \mathcal{H}: x^{\prime} \in \operatorname{Ran}(\mathbf{T})\right\} .
$$

The subspace

$$
\mathbf{T}(0)=\left\{x^{\prime} \in \mathcal{H}:\left\langle 0, x^{\prime}\right\rangle \in \mathbf{T}\right\}
$$

is called the multi-valued part of $\mathbf{T}$. A subspace $\mathbf{T} \ominus\langle 0, \mathbf{T}(0)\rangle$ is the graph of a linear operator $T, \operatorname{Dom}(T)=\operatorname{Dom}(\mathbf{T})$, which is called the operator part of $\mathbf{T}$. Clearly, $\mathbf{T} x=T x \oplus \mathbf{T}(0)$. The adjoint $\mathbf{T}^{*}$ to $\mathbf{T}$ is given by

$$
\mathbf{T}^{*}=\mathcal{H}^{2} \ominus\left\{\left\langle-x^{\prime}, x\right\rangle,\left\langle x, x^{\prime}\right\rangle \in \mathbf{T}\right\} .
$$

The numerical range of a 1.r. $\mathbf{T}$ is the set

$$
W(\mathbf{T})=\{(\mathbf{T} x, x), x \in \operatorname{Dom}(\mathbf{T}),\|x\|=1\} .
$$

As has been shown in [47] if $W(\mathbf{T}) \neq \mathbb{C}$, then $\mathbf{T}(0) \subseteq \mathcal{H} \ominus \overline{\operatorname{Dom}}(\mathbf{T})$.

A 1.r. $\mathbf{T}$ is called

- Hermitian if $W(\mathbf{T}) \subseteq \mathbb{R} \Longleftrightarrow \mathbf{T} \subseteq \mathbf{T}^{*}$;

- $\quad$ selfadjoint if $\mathbf{T}=\mathbf{T}^{*}$;

- nonnegative if $W(\mathbf{T}) \subseteq \mathbb{R}_{+}$;

- accretive if $W(\mathbf{T}) \subseteq \Pi_{+}$;

- $m$-accretive if $\mathbf{T}$ is accretive and has no accretive extensions in $\mathcal{H}^{2}$;

- $\alpha$-sectorial if $W(\mathbf{T}) \subseteq \mathcal{S}(\alpha)$;

- $m$ - $\alpha$-sectorial if $\mathbf{T}$ is $\alpha$-sectorial and $m$-accretive.

It is well-known that is there is one-to-one correspondence between all m-accretive 1.r. $\mathbf{U}$ in a Hilbert space $\mathcal{H}$ and all contractions $\mathcal{U}$ in $\mathcal{H}$ given by fractional-linear transformations

$$
\begin{aligned}
\mathbf{U} & =\{\langle(I+\mathcal{U}) h,(I-\mathcal{U}) h\rangle, h \in \mathcal{H}\}=(I-\mathcal{U})(I+\mathcal{U})^{-1} \\
\mathcal{U}\left(x+x^{\prime}\right) & =x-x^{\prime},\left\langle x, x^{\prime}\right\rangle \in \mathbf{U} \Longleftrightarrow \mathcal{U}=(I-\mathbf{U})(I+\mathbf{U})^{-1}
\end{aligned}
$$

Moreover [3]

$$
\mathcal{U} \in C_{\mathcal{H}}(\alpha) \Longleftrightarrow \mathbf{U}=(I-\mathcal{U})(I+\mathcal{U})^{-1} \text { is } \mathrm{m} \text { - } \alpha \text {-sectorial l.r.. }
$$


In particular, a l.r. $\mathbf{U}$ is self-adjoint and nonnegative if and only if the operator $\mathcal{U}$ is self-adjoint contraction.

\subsection{Sectorial Sesquilinear Forms}

Recall some definitions and results from [35]. Let $\tau[\cdot, \cdot]$ be a sesquilinear form in a Hilbert space $\mathcal{H}$ defined on a linear manifold Dom $(\tau)$. The form $\tau$ is called symmetric if $\tau[u, v]=\overline{\tau[v, u]}$ for all $u, v \in \operatorname{Dom}(\tau)$ and nonnegative if $\tau[u]:=\tau[u, u] \geq 0$ for all $u \in \operatorname{Dom}(\tau)$.

The form $\tau$ is called sectorial with the vertex at the point $\gamma \in \mathbb{C}$ and a semi-angle $\alpha \in[0, \pi / 2)$ if its numerical range

$$
W(\tau)=\{\tau[u], u \in \operatorname{Dom}(\tau),\|u\|=1\}
$$

is contained in the sector $\{z \in \mathbb{C}:|\arg (z-\gamma)| \leq \alpha\}$, i.e.,

$$
\left|\operatorname{Im}\left(\tau[u]-\gamma\|u\|^{2}\right)\right| \leq \tan \alpha \operatorname{Re}\left(\tau[u]-\gamma\|u\|^{2}\right), u \in \operatorname{Dom}(\tau)
$$

Thus, $\tau$ is sectorial with vertex at $\gamma$ if and only if the form $\tau[u, v]-\gamma(u, v)$ has vertex at the origin.

Let $\tau$ be a sesquilinear form. The form $\tau^{*}[u, v]:=\overline{\tau[v, u]}$ is called the adjoint to $\tau$, and the forms

$$
\begin{aligned}
& \tau_{\mathrm{R}}[u, v]:=\frac{1}{2}\left(\tau[u, v]+\tau^{*}[u, v]\right), \\
& \tau_{\mathrm{I}}[u, v]:=\frac{1}{2 i}\left(\tau[u, v]-\tau^{*}[u, v]\right), u, v \in \operatorname{Dom}(\tau)
\end{aligned}
$$

are called the real and the imaginary parts of $\tau$, respectively.

A sequence $\left\{u_{n}\right\}$ is called $\tau$-converging to the vector $u \in \mathcal{H}$ if

$$
\lim _{n \rightarrow \infty} u_{n}=u \text { and } \lim _{n, m \rightarrow \infty} \tau\left[u_{n}-u_{m}\right]=0 .
$$

The form $\tau$ is called closed if for every sequence $\left\{u_{n}\right\} \tau$-converging to a vector $u$ it follows that $u \in \operatorname{Dom}(\tau)$ and $\lim _{n \rightarrow \infty} \tau\left[u-u_{n}\right]=0$. A sectorial form $\tau$ with vertex at the origin is closed if and only if the linear manifold Dom $(\tau)$ is a Hilbert space with the inner product $(u, v)_{\tau}=\tau_{\mathrm{R}}[u, v]+(u, v)$ [35]. The form $\tau$ is called closable if it has a closed extension; in this case the closure of $\tau$ is the smallest closed extension of $\tau$. If $\tau$ is a closed, densely defined sectorial form, then according to First Representation Theorem [35,37] there exists a unique m-sectorial operator $T$ in $\mathcal{H}$, associated with $\tau$, i.e.,

$$
(T u, v)=\tau[u, v] \text { for all } u \in \operatorname{Dom}(T) \text { and for all } v \in \operatorname{Dom}(\tau)
$$


In this case the operator $T^{*}$ is associated with the adjoint form

$$
\tau^{*}[u, v]=\overline{\tau[v, u]}, u, v \in \operatorname{Dom}(\tau)
$$

The nonnegative self-adjoint operator, denoted by $T_{R}$, associated with the real part

$$
\tau_{R}[u, v]=\frac{1}{2}\left(\tau[u, v]+\tau^{*}[u, v]\right), \quad u, v \in \operatorname{Dom}(\tau)
$$

of the form $\tau$ is called the "real part" of $T$. According to Second Representation Theorem [35,37] the identities hold:

$$
\operatorname{Dom}(\tau)=\operatorname{Dom}\left(T_{R}^{\frac{1}{2}}\right), \tau_{R}[u, v]=\left(T_{R}^{\frac{1}{2}} u, T_{R}^{\frac{1}{2}} v\right) .
$$

If the form $\tau$ is $\alpha$-sectorial, then it has the representation

$$
\tau[u, v]=\left((I+i M) T_{R}^{\frac{1}{2}} u, T_{R}^{\frac{1}{2}} v\right), u, v \in \operatorname{Dom}(\tau),
$$

where $M$ is a bounded self-adjoint operator in the subspace $\overline{\operatorname{Ran}}\left(T_{R}\right)$ and $\|M\| \leq$ $\tan \alpha$. For $T$ one obtains

$\operatorname{Dom}(T)=\left\{u \in \operatorname{Dom}(\tau):(I+i M) T_{R}^{1 / 2} u \in \operatorname{Dom}(\tau)\right\}, T=T_{R}^{1 / 2}(I+i M) T_{R}^{1 / 2} u$.

If $T$ is a sectorial operator, then the form

$$
\tau[u, v]=(T u, v), \quad u, v \in \operatorname{Dom}(T)
$$

is closable. The domain of its closure $T[\cdot, \cdot]$ we denote by $\mathcal{D}[T]$.

If $\tau$ is closed but non-densely defined sectorial form in the Hilbert space $\mathcal{H}$, then with $\tau$ is associated the $m$-sectorial linear relation $\mathbf{T}$ [47]. Moreover,

$$
\begin{aligned}
(\mathbf{T} x, y)_{\mathcal{H}} & =(T x, y)_{\mathcal{H}}, x, y \in \operatorname{Dom}(\mathbf{T}), \\
\operatorname{Dom}(\tau) & =\mathcal{D}[\mathbf{T}]=\mathcal{D}[T],
\end{aligned}
$$

where $T$ is the operator part of $\mathbf{T}$. Let $\mathbf{T}_{1}$ and $\mathbf{T}_{2}$ be two nonnegative self-adjoint linear relations. We write $\mathbf{T}_{1} \leq \mathbf{T}_{2}$ if

$$
\mathcal{D}\left[\mathbf{T}_{1}\right] \supseteq \mathcal{D}\left[\mathbf{T}_{2}\right] \text { and } \mathbf{T}_{1}[u] \leq \mathbf{T}_{2}[u], u \in \mathcal{D}\left[\mathbf{T}_{2}\right]
$$

The next theorem will be used in Sect. 3 .

Theorem 2.1 Let a sesquilinear form $\tau[u, v]$ be nonnegative and closed in the Hilbert space $\mathcal{H}$ with the inner product $(\cdot, \cdot)_{\mathcal{H}}$. Let $\mathbf{T}$ be the associated nonnegative selfadjoint linear relation in $\mathcal{H}$ and let $\mathcal{T}$ be its fractional-linear transformation $\mathcal{T}=$ $(I-\mathbf{T})(I+\mathbf{T})^{-1}$. 
A m-accretive l.r. $\mathbf{U}$ satisfies the condition

$$
\operatorname{Ran}(\mathbf{U}) \subset \operatorname{Dom}(\tau), \operatorname{Re}(\mathbf{U} x, x)_{\mathcal{H}} \geq \tau[\mathbf{U} x], x \in \operatorname{Dom}(\mathbf{U})
$$

if and only if the fractional-linear transformation $\mathcal{U}$ of $\mathbf{U}$ has the representation

$$
\mathcal{U}=I-\frac{1}{2}(I+\mathcal{T})^{1 / 2}(I+\mathcal{Y})(I+\mathcal{T})^{1 / 2},
$$

where $\mathcal{Y}$ is a contraction in the subspace $\overline{\operatorname{Dom}}(\tau)=\overline{\operatorname{Ran}}(I+\mathcal{T})$.

Proof Let $x=(I+\mathcal{U}) h, x^{\prime}=(I-\mathcal{U}) h$, where $h \in \mathcal{H}$. Then $\left\langle x, x^{\prime}\right\rangle \in \mathbf{U}$ and

$$
\begin{aligned}
\left(x^{\prime}, x\right)_{\mathcal{H}} & =(\mathbf{U} x, x)_{\mathcal{H}}=((I-\mathcal{U}) h,(I+\mathcal{U}) h)_{\mathcal{H}} \\
& =-\|(I-\mathcal{U}) h\|_{\mathcal{H}}^{2}+2((I-\mathcal{U}) h, h)_{\mathcal{H}} \\
& =-\left\|x^{\prime}\right\|_{\mathcal{H}}^{2}+2((I-\mathcal{U}) h, h)_{\mathcal{H}} .
\end{aligned}
$$

Similarly for $y=(I+\mathcal{T}) h$ and $y^{\prime}=(I-\mathcal{T}) h$ we have $\left\langle y, y^{\prime}\right\rangle \in \mathbf{T}$ and

$$
\left(y^{\prime}, y\right)_{\mathcal{H}}=(\mathbf{T} y, y)_{\mathcal{H}}=-\|y\|_{\mathcal{H}}^{2}+2\left\|(I+\mathcal{T})^{1 / 2} h\right\|_{\mathcal{H}}^{2} .
$$

Passing to the closure, we obtain

$$
\begin{aligned}
\operatorname{Dom}(\tau) & =\operatorname{Ran}\left((I+\mathcal{T})^{1 / 2}\right), \\
\tau[v] & =-\|v\|_{\mathcal{H}}^{2}+2\left\|(I+\mathcal{T})^{-1 / 2} v\right\|_{\mathcal{H}}^{2}, v \in \operatorname{Dom}(\tau),
\end{aligned}
$$

where $(I+\mathcal{T})^{-1}$ is Moore-Penrose inverse for $(I+\mathcal{T})$.

Hence

$$
\operatorname{Ran}(\mathbf{U}) \subset \operatorname{Dom}(\tau) \Longleftrightarrow \operatorname{Ran}(I-\mathcal{U}) \subset \operatorname{Ran}\left((I+\mathcal{T})^{1 / 2}\right)
$$

and

$$
(\mathbf{U} x, x)_{\mathcal{H}}-\tau[\mathbf{U} x]=2((I-\mathcal{U}) h, h)_{\mathcal{H}}-2\left\|(I+\mathcal{T})^{-1 / 2}(I-\mathcal{U}) h\right\|_{\mathcal{H}}^{2}
$$

for $x=(I+\mathcal{U}) h, \mathbf{U} x=x^{\prime}=(I-\mathcal{U}) h, h \in \mathcal{H}$.

Suppose that $\operatorname{Re}(\mathbf{U} x, x)_{\mathcal{H}} \geq \tau[\mathbf{U} x]$ for all $x \in \operatorname{Dom}(\mathbf{U})$. Then

$$
\operatorname{Re}((I-\mathcal{U}) h, h)_{\mathcal{H}} \geq\left\|(I+\mathcal{T})^{-1 / 2}(I-\mathcal{U}) h\right\|_{\mathcal{H}}^{2} \quad \text { for all } \quad h \in \mathcal{H}
$$

Let $\mathcal{U}_{R}=\left(\mathcal{U}+\mathcal{U}^{*}\right) / 2$ be the real part of $\mathcal{U}$. Then (2.9) yields the equality

$$
(I+\mathcal{T})^{-1 / 2}(I-\mathcal{U})=\mathcal{V}\left(I-\mathcal{U}_{R}\right)^{1 / 2}
$$


where $\mathcal{V}: \overline{\operatorname{Ran}}\left(I-\mathcal{U}_{R}\right) \rightarrow \overline{\operatorname{Ran}}(I+\mathcal{T})$ is a contraction. It follows that

$$
I-\mathcal{U}=(I+\mathcal{T})^{1 / 2} \mathcal{V}\left(I-\mathcal{U}_{R}\right)^{1 / 2}
$$

and for all $h \in \mathcal{H}$

$$
\begin{aligned}
\left\|\left(I-\mathcal{U}_{R}\right)^{1 / 2} h\right\|_{\mathcal{H}}^{2} & =\operatorname{Re}((I-\mathcal{U}) h, h)_{\mathcal{H}}=\operatorname{Re}\left((I+\mathcal{T})^{1 / 2} h, \mathcal{V}\left(I-\mathcal{U}_{R}\right)^{1 / 2} h\right)_{\mathcal{H}} \\
& \leq\left\|(I+\mathcal{T})^{1 / 2} h\right\|_{\mathcal{H}}\left\|\left(I-\mathcal{U}_{R}\right)^{1 / 2} h\right\|_{\mathcal{H}} \cdot
\end{aligned}
$$

Therefore $\left\|\left(I-\mathcal{U}_{R}\right)^{1 / 2} h\right\|_{\mathcal{H}} \leq\left\|(I+\mathcal{T})^{1 / 2} h\right\|_{\mathcal{H}}$ and, as a consequence, for all $h, g \in \mathcal{H}$

$$
\begin{aligned}
\left|((I-\mathcal{U}) h, g)_{\mathcal{H}}\right| & =\left|\left(\mathcal{V}\left(I-\mathcal{U}_{R}\right)^{1 / 2} h,(I+\mathcal{T})^{1 / 2} g\right)_{\mathcal{H}}\right| \\
& \leq\left\|\left(I-\mathcal{U}_{R}\right)^{1 / 2} h\right\|_{\mathcal{H}}\left\|(I+\mathcal{T})^{1 / 2} g\right\|_{\mathcal{H}} \\
& \leq\left\|(I+\mathcal{T})^{1 / 2} h\right\|_{\mathcal{H}}\left\|(I+\mathcal{T})^{1 / 2} g\right\|_{\mathcal{H}} .
\end{aligned}
$$

It follows that

$$
I-\mathcal{U}=(I+\mathcal{T})^{1 / 2} \mathcal{Z}(I+\mathcal{T})^{1 / 2},
$$

where $\mathcal{Z}$ is a contraction in the subspace $\mathcal{H}_{0}:=\overline{\operatorname{Ran}}(I+\mathcal{T})$. This equality produces for all $h \in \mathcal{H}$

$$
\operatorname{Re}((I-\mathcal{U}) h, h)_{\mathcal{H}}=\operatorname{Re}\left(\mathcal{Z}(I+\mathcal{T})^{1 / 2} h,(I+\mathcal{T})^{1 / 2} h\right)_{\mathcal{H}},
$$

and by (2.9)

$$
\operatorname{Re}\left(\mathcal{Z}(I+\mathcal{T})^{1 / 2} h,(I+\mathcal{T})^{1 / 2} h\right)_{\mathcal{H}} \geq\left\|\mathcal{Z}(I+\mathcal{T})^{1 / 2} h\right\|_{\mathcal{H}}^{2}
$$

Therefore

$$
\operatorname{Re}(\mathcal{Z} \varphi, \varphi)_{\mathcal{H}} \geq\|\mathcal{Z} \varphi\|_{\mathcal{H}}^{2} \text { for all } \varphi \in \mathcal{H}_{0}
$$

Let $\mathcal{Y}=2 \mathcal{Z}-I$. Then $\mathcal{Z}=(I+\mathcal{Y}) / 2$. Because

$2 \operatorname{Re}((I+\mathcal{Y}) \varphi, \varphi)_{\mathcal{H}}-\|(I+\mathcal{Y}) \varphi\|_{\mathcal{H}}^{2}=\|\varphi\|_{\mathcal{H}}^{2}-\|\mathcal{Y} \varphi\|_{\mathcal{H}}^{2}, \varphi \in \mathcal{H}_{0}$,

(2.11) yields that $\mathcal{Y}$ is a contraction in $\mathcal{H}_{0}$ and

$$
\mathcal{U}=I-\frac{1}{2}(I+\mathcal{T})^{1 / 2}(I+\mathcal{Y})(I+\mathcal{T})^{1 / 2} .
$$


Conversely, suppose that an operator $\mathcal{U}$ takes the form (2.7) with some contraction $\mathcal{Y}$ in $\mathcal{H}_{0}$. Let us prove that $\mathcal{U}$ is a contraction in $\mathcal{H}$. Because of

$$
2 \operatorname{Re}((I-\mathcal{U}) h, h)_{\mathcal{H}}-\|(I-\mathcal{U}) h\|_{\mathcal{H}}^{2}=\|h\|_{\mathcal{H}}^{2}-\|\mathcal{U} h\|_{\mathcal{H}}^{2}, h \in \mathcal{H}
$$

it is sufficient to proof that $2 \operatorname{Re}((I-\mathcal{U}) h, h)_{\mathcal{H}}-\|(I-\mathcal{U}) h\|_{\mathcal{H}}^{2} \geq 0$ for all $h \in \mathcal{H}$. Denote $\varphi=(I+\mathcal{T})^{1 / 2} h$. By (2.7) we have

$$
\begin{aligned}
2 \operatorname{Re} & ((I-\mathcal{U}) h, h)_{\mathcal{H}}-\|(I-\mathcal{U}) h\|_{\mathcal{H}}^{2}=\operatorname{Re}((I+\mathcal{Y}) \varphi, \varphi)_{\mathcal{H}} \\
& -\frac{1}{4}\left\|(I+\mathcal{T})^{1 / 2}(I+\mathcal{Y}) \varphi\right\|_{\mathcal{H}}^{2}=\operatorname{Re}((I+\mathcal{Y}) \varphi, f)_{\mathcal{H}} \\
& -\frac{1}{4}\|(I+\mathcal{Y}) \varphi\|_{\mathcal{H}}^{2}-\frac{1}{4}(\mathcal{T}(I+\mathcal{Y}) \varphi,(I+\mathcal{Y}) f)_{\mathcal{H}} \\
= & \operatorname{Re}((I+\mathcal{Y}) \varphi, f)_{\mathcal{H}}-\frac{1}{2}\|(I+\mathcal{Y}) \varphi\|_{\mathcal{H}}^{2} \\
& +\frac{1}{4}\|(I+\mathcal{Y}) \varphi\|_{\mathcal{H}}^{2}-\frac{1}{4}(\mathcal{T}(I+\mathcal{Y}) \varphi,(I+\mathcal{Y}) f)_{\mathcal{H}} \\
= & \|\varphi\|_{\mathcal{H}}^{2}-\|\mathcal{Y} \varphi\|_{\mathcal{H}}^{2}+\frac{1}{4}\left\|(I-\mathcal{T})^{1 / 2}(I+\mathcal{Y}) \varphi\right\|_{\mathcal{H}}^{2} \geq 0 .
\end{aligned}
$$

Thus the operator $\mathcal{U}$ is contraction in $\mathcal{H}$. Moreover, $\operatorname{Ran}(I-\mathcal{U}) \subset \operatorname{Ran}\left((I+\mathcal{T})^{1 / 2}\right)=$ $\operatorname{Dom}(\tau)$ and

$$
(I+\mathcal{T})^{-1 / 2}(I-\mathcal{U})=\frac{1}{2}(I+\mathcal{Y})(I+\mathcal{T})^{1 / 2}
$$

Denoting again $\varphi=(I+\mathcal{T})^{1 / 2} h$, where $h \in \mathcal{H}$ we obtain

$$
\begin{aligned}
\operatorname{Re}((I-\mathcal{U}) h, h)_{\mathcal{H}} & \geq\left\|(I+\mathcal{T})^{-1 / 2}(I-\mathcal{U}) h\right\|_{\mathcal{H}}^{2} \\
& =\frac{1}{2} \operatorname{Re}((I+\mathcal{Y}) \varphi, \varphi)_{\mathcal{H}}-\frac{1}{4}\|(I+\mathcal{Y}) \varphi\|_{\mathcal{H}}^{2} \\
& =\frac{1}{4}\left(\|\varphi\|_{\mathcal{H}}^{2}-\|\mathcal{Y} \varphi\|_{\mathcal{H}}^{2}\right) \geq 0 .
\end{aligned}
$$

Thus, holds (2.9). Let $\mathbf{U}=\{\langle(I+\mathcal{U}) h,(I-\mathcal{U}) h\rangle, h \in \mathcal{H}\}$. Since $\mathcal{U}$ is a contraction, the linear relation $\mathbf{U}$ is $m$-accretive, $\operatorname{Ran}(\mathbf{U}) \subset \operatorname{Dom}(\tau)$, and

$$
\operatorname{Re}(\mathbf{U} x, x)_{\mathcal{H}} \geq \tau[\mathbf{U} x], x \in \operatorname{Dom}(\mathbf{U})
$$

holds.

Corollary 2.2 A m-accretive l.r. $\mathbf{U}$ in $\mathcal{H}$ satisfies the condition

$$
\begin{aligned}
& \operatorname{Ran}(\mathbf{U}) \subset \operatorname{Dom}(\tau), \\
& \tan \alpha\left(\operatorname{Re}(\mathbf{U} x, x)_{\mathcal{H}}-\tau[\mathbf{U} x]\right) \geq\left|\operatorname{Im}(\mathbf{U} x, x)_{\mathcal{H}}\right|, x \in \operatorname{Dom}(\mathbf{U})
\end{aligned}
$$


if and only if the fractional-linear transformation $\mathcal{U}$ of $\mathbf{U}$ has the representation (2.7) with $\mathcal{Y}$ satisfying the condition

$$
\|\mathcal{Y} \sin \alpha \pm i \cos \alpha I\|_{\mathcal{H}} \leq 1
$$

i.e., $\mathcal{Y} \in C_{\mathcal{H}_{0}}(\alpha)$, where $\mathcal{H}_{0}=\overline{\operatorname{Dom}}(\tau)$.

Proof Condition (2.13) is equivalent to the following

$$
\tan \alpha\left(\|\varphi\|_{\mathcal{H}}^{2}-\|\mathcal{Y} \varphi\|_{\mathcal{H}}^{2}\right) \geq 2\left|\operatorname{Im}(\mathcal{Y} \varphi, \varphi)_{\mathcal{H}}\right|, \varphi \in \operatorname{Dom}(\mathcal{Y})
$$

From (2.8) and (2.7) it follows that (2.12) is equivalent to

$$
\tan \alpha\left(\operatorname{Re}((I+\mathcal{Y}) \varphi, \varphi)_{\mathcal{H}}-\frac{1}{2}\|(I+\mathcal{Y}) \varphi\|_{\mathcal{H}}^{2}\right) \geq\left|\operatorname{Im}((I+\mathcal{Y}) \varphi, \varphi)_{\mathcal{H}}\right|
$$

for all $\varphi \in \mathcal{H}_{0}=\operatorname{Dom}(\mathcal{Y})$. The right hand side of the above inequality is exactly

$$
\frac{1}{2}\left(\|\varphi\|_{\mathcal{H}}^{2}-\|\mathcal{Y} \varphi\|_{\mathcal{H}}^{2}\right)
$$

Thus U satisfies (2.12) iff (2.13) holds.

Remark 2.3 In [17] for nonnegative self-adjoint 1.r. $\mathbf{U}$ and $\mathbf{T}$ it is proved that the following statements

(i) $\operatorname{Ran}(\mathbf{U}) \subset \operatorname{Dom}(\mathbf{T})$ and $(\mathbf{U} u, u) \geq \mathbf{T}[\mathbf{U} u], u \in \operatorname{Dom}(\mathbf{U})$,

(ii) $\mathbf{U} \geq \mathbf{T}^{-1}$,

(iii) $\mathbf{U}^{-1} \leq \mathbf{T}$

are equivalent.

If $\mathbf{U}$ is a 1.r. and $\mathbf{T}$ is a nonnegative 1.r., then one can easily prove that the statements

(i) $\operatorname{Ran}(\mathbf{U}) \subset \operatorname{Dom}(\mathbf{T})$ and $\operatorname{Re}(\mathbf{U} u, u) \geq \mathbf{T}[\mathbf{U} u]$,

(ii) $\operatorname{Re}\left(\mathbf{U}^{-1} x, x\right) \geq \mathbf{T}[x], x \in \operatorname{Ran}(\mathbf{U})$

are equivalent.

\subsection{Quasi-Self-Adjoint Extensions of Symmetric Operator}

Let $\mathfrak{H}$ be a separable complex Hilbert space and let $S$ be a symmetric operator in $\mathfrak{H}$. Let

$$
\mathfrak{N}_{z}=\mathfrak{H} \ominus(S-\bar{z} I)=\operatorname{Ker}\left(S^{*}-z I\right), \quad \operatorname{Im} z \neq 0
$$

be the defect subspace of $S$. The numbers $n_{ \pm}=\operatorname{dim} \mathfrak{N}_{ \pm i}$ are called the defect numbers of $S$. By well-known J. von Neumann's formula the direct decomposition

$$
\operatorname{Dom}\left(S^{*}\right)=\operatorname{Dom}(S) \dot{+} \mathfrak{N}_{z} \dot{+} \mathfrak{N}_{\bar{z}}, \quad \operatorname{Im} z \neq 0
$$


holds. We consider the domain Dom $\left(S^{*}\right)$ of the adjoint $S^{*}$ to $S$ as the Hilbert $\mathfrak{H}_{+}$ space with the inner product

$$
(u, v)_{+}=(u, v)+\left(S^{*} u, S^{*} v\right)
$$

Then holds (+)-orthogonal decomposition:

$$
\mathfrak{H}_{+}=\operatorname{Dom}(S) \oplus \mathfrak{N}_{i} \oplus \mathfrak{N}_{-i} .
$$

Extensions $T$ of $S$ possessing property

$$
S \subset T \subset S^{*}
$$

are called quasi-self-adjoint (proper, intermediate) extensions of $S$.

Let

$$
\mathfrak{L}:=\mathfrak{N}_{i} \oplus \mathfrak{N}_{-i}
$$

Then

$$
S^{* 2} f=-f,\left(S^{*} f, S^{*} g\right)_{+}=(f, g)_{+}, f, g \in \mathfrak{L} .
$$

The next statement is well-known.

Theorem 2.4 The formulas

$$
\operatorname{Dom}(T)=\operatorname{Dom}(S) \oplus \mathfrak{K}, T=S^{*} \uparrow \operatorname{Dom}(T)
$$

give a one-to-one correspondence between subspaces $\mathfrak{K} \subset \mathfrak{N}_{i} \oplus \mathfrak{N}_{-i}$ and quasi-selfadjoint extensions $T$ of $S$. The adjoint operator $T^{*}$ is given by

$$
\operatorname{Dom}\left(T^{*}\right)=\operatorname{Dom}(S) \oplus S^{*} \mathfrak{K}^{\perp}, T^{*}=S^{*} \uparrow \operatorname{Dom}\left(T^{*}\right),
$$

where $\mathfrak{K}^{\perp}:=\mathfrak{L} \ominus \mathfrak{K}$ is $(+)$-orthogonal complement to $\mathfrak{K}$ in $\mathfrak{L}$.

In particular, a maximal dissipative (anti-dissipative) extension $T$ of $S$ is quasiself-adjoint and

$\operatorname{Dom}(T)=\operatorname{Dom}(S) \oplus(I-M) \mathfrak{N}_{i} \quad\left(\operatorname{Dom}(T)=\operatorname{Dom}(S) \oplus(I-M) \mathfrak{N}_{-i}\right)$,

$\operatorname{Dom}\left(T^{*}\right)=\operatorname{Dom}(S) \oplus\left(I-M^{*}\right) \mathfrak{N}_{-i} \quad\left(\operatorname{Dom}\left(T^{*}\right)=\operatorname{Dom}(S) \oplus\left(I-M^{*}\right) \mathfrak{N}_{i}\right)$,

where $M \in \mathbf{L}\left(\mathfrak{N}_{i}, \mathfrak{N}_{-i}\right)\left(\mathbf{L}\left(\mathfrak{N}_{-i}, \mathfrak{N}_{i}\right)\right)$ is a contraction in $\mathfrak{H}\left(\mathfrak{H}_{+}\right)$and $M^{*} \in$ $\mathbf{L}\left(\mathfrak{N}_{-i}, \mathfrak{N}_{i}\right)\left(\mathbf{L}\left(\mathfrak{N}_{i}, \mathfrak{N}_{-i}\right)\right)$ denotes its $(+)$-adjoint. 
According to J. von Neumann the operator $S$ has self-adjoint extensions in $\mathfrak{H}$ if and only if defect numbers of $S$ are equal. In this case the domain of any self-adjoint extension $A$ of $S$ takes the form

$$
\operatorname{Dom}(A)=\operatorname{Dom}(S) \oplus(I-V) \mathfrak{N}_{i}
$$

where $V$ is an isometric operator in $\mathfrak{H}\left(\mathfrak{H}_{+}\right)$from $\mathfrak{N}_{i}$ onto $\mathfrak{N}_{-i}$. Fix a self-adjoint extension $A$ of $S$ and put

$$
\mathfrak{N}_{A}=(I-V) \mathfrak{N}_{i}, \mathfrak{M}_{A}=(I+V) \mathfrak{N}_{i}
$$

where $V$ is the corresponding isometry from $\mathfrak{N}_{i}$ onto $\mathfrak{N}_{-i}$. Then the following relations hold:

$$
\begin{aligned}
\mathfrak{M}_{A} & =A \mathfrak{N}_{A},(A+i I) \mathfrak{N}_{A}=\mathfrak{N}_{i},(A-i I) \mathfrak{N}_{A}=\mathfrak{N}_{-i}, \\
\mathfrak{N}_{A} & =\left\{f \in \operatorname{Dom}(A): S^{*} A f=-f\right\}, \\
\mathfrak{M}_{A} & =\left\{f \in \operatorname{Dom}\left(S^{*}\right): A S^{*} f=-f\right\}, \\
\operatorname{Dom}(A) & =\operatorname{Dom}(S) \oplus \mathfrak{N}_{A}, \\
\mathfrak{H}_{+} & =\operatorname{Dom}(S) \oplus \mathfrak{N}_{A} \oplus \mathfrak{M}_{A} .
\end{aligned}
$$

A quasi-self-adjoint extension $T$ is called relatively prime (or disjoint) with $A$ if

$$
\operatorname{Dom}(T) \cap \operatorname{Dom}(A)=\operatorname{Dom}(S)
$$

and transversal to $A$ if

$$
\operatorname{Dom}(T)+\operatorname{Dom}(A)=\operatorname{Dom}\left(S^{*}\right) .
$$

The part of following Proposition related to self-adjoint extensions is proved in [15].

\section{Proposition 2.5 The formulas}

$$
\begin{aligned}
\operatorname{Dom}(T) & =\operatorname{Dom}(S) \oplus(A+C) \operatorname{Dom}(C), \\
T\left(f_{0}+(A+C) h\right) & =A\left(f_{0}+C h\right)-h, f_{0} \in \operatorname{Dom}(S), h \in \operatorname{Dom}(C)
\end{aligned}
$$

give a one-to-one correspondence between quasi-self-adjoint extensions $T$ of $S$ relatively prime with $A$ and closed operators $C$ in $\mathfrak{N}_{A}$.

The extension $T$ is transversal to $A$ if and only if $\operatorname{Dom}(C)=\mathfrak{N}_{A}$.

The extension $T$ is self-adjoint if and only if $C$ is self-adjoint operator in $\mathfrak{N}_{A}$.

The extension $T$ is maximal dissipative if and only if $C$ is maximal dissipative operator in $\mathfrak{N}_{A}$.

Proof The closed operator $C: \mathfrak{N}_{A} \rightarrow \mathfrak{N}_{A}$ can be represented as

$$
C(I-V) f_{i}=(I-V) U f_{i}, f_{i} \in \operatorname{Dom}(U) \subset \mathfrak{N}_{i},
$$


where $U$ is a closed operator. Then

$$
\begin{aligned}
(A+C)(I-V) f_{i} & =i(I+V) f_{i}+(I-V) U f_{i} \\
& =(i I+U) f_{i}+V(i I-U) f_{i}, f_{i} \in \mathfrak{N}_{i} .
\end{aligned}
$$

It follows that $T$ is maximal dissipative if and only if the operator $M:=(U-i I)(i I+$ $U)^{-1}$ is well defined on whole $\mathfrak{N}_{i}$ and is a contraction or equivalently, the operator $U$ is maximal dissipative in $\mathfrak{N}_{i}$. The last is equivalent to $U$ is the maximal dissipative operator in $\mathfrak{N}_{A} \subset \mathfrak{H}_{+}$.

Proposition 2.6 Let $U$ be a $(+)$-closed and densely defined operator in $\mathfrak{N}_{A}$. Then the operator $T$ given by

$$
\begin{aligned}
\operatorname{Dom}(T) & =\operatorname{Dom}(S) \oplus(I+A U) \operatorname{Dom}(U), \\
T\left(f_{0}+(I+A U) h\right) & =A\left(f_{0}+h\right)-U h, f_{0} \in \operatorname{Dom}(S), h \in \operatorname{Dom}(U)
\end{aligned}
$$

is a quasi-self-adjoint extension of $S$. Its adjoint $T^{*}$ is of the form

$$
\begin{aligned}
& \operatorname{Dom}\left(T^{*}\right)=\operatorname{Dom}(S) \oplus\left(I+A U^{*}\right) \operatorname{Dom}\left(U^{*}\right), \\
& T^{*}\left(f_{0}+\left(I+A U^{*}\right) e\right)=A\left(f_{0}+e\right)-U^{*} e, \\
& f_{0} \in \operatorname{Dom}(S), e \in \operatorname{Dom}\left(U^{*}\right),
\end{aligned}
$$

where $U^{*}$ is $(+)$-adjoint of $U$ in $\mathfrak{N}_{A}$. In this case the extension $T$ is relatively prime with $A$ if and only if $\operatorname{Ker} U=\{0\}$ and is transversal to $A$ if and only if the number 0 is the regular number of $U$, i.e. $\operatorname{Ker} U=\{0\}$ and $\operatorname{Ran}(U)=\mathfrak{N}_{A}$.

Proof Let us find the orthogonal complement $\mathfrak{L} \ominus(I+A U) \operatorname{Dom}(U)$. Let $\varphi \in \mathfrak{L}$. Then

$$
\varphi=\varphi_{1}+\varphi_{2}, \varphi_{1} \in \mathfrak{N}_{A}, \varphi_{2} \in \mathfrak{M}_{A}=S^{*} \mathfrak{N}_{A}
$$

Then for all $f \in \operatorname{Dom}(U)$, using (2.16), we have

$$
((I+A U) f, \varphi)_{+}=\left(f, \varphi_{1}\right)_{+}+\left(A U f, \varphi_{2}\right)_{+}=\left(f, \varphi_{1}\right)_{+}-\left(U f, S^{*} \varphi_{2}\right)_{+} .
$$

Put $h=S^{*} \varphi_{2}$ then $h \in \mathfrak{N}_{A}$ and $\varphi_{2}=-S^{*} h=-A h$. Then

$((I+A U) f, \varphi)_{+}=0$ for all $f \in \operatorname{Dom}(U) \Longleftrightarrow h \in \operatorname{Dom}\left(U^{*}\right)$ and $\varphi_{1}=U^{*} h$.

Thus

$$
\begin{aligned}
\mathfrak{L} \ominus(I+A U) \operatorname{Dom}(U) & =\left(U^{*}-A\right) \operatorname{Dom}\left(U^{*}\right), \\
S^{*}(\mathfrak{L} \ominus(I+A U) \operatorname{Dom}(U)) & =\left(I+A U^{*}\right) \operatorname{Dom}\left(U^{*}\right) .
\end{aligned}
$$

Now relations (2.22) follow from (2.4). 
2.7 Nonnegative Self-Adjoint Extensions of a Nonnegative Symmetric Operator

Let $S$ be a nonnegative symmetric operator. Then the defect numbers of $S$ are equal and therefore $S$ admits self-adjoint extensions.

Recall the definition of the Friedrichs extension of $S$ [35]. Let $S[\cdot, \cdot]$ the closure of the sesquilinear form $(S f, g), f, g \in \operatorname{Dom}(S)$. According to First Representation Theorem there exists a nonnegative self-adjoint operator $S_{F}$ associated with $S[\cdot, \cdot]$, i.e.

$$
\left(S_{F} u, v\right)=S[u, v], v \in \mathcal{D}[S], u \in \operatorname{Dom}\left(S_{F}\right) .
$$

The operator $S_{F}$ is a self-adjoint extension of $S$ and is called the Friedrichs extension of $S$. Note that

$$
\operatorname{Dom}\left(S_{F}\right)=\mathcal{D}[S] \cap \operatorname{Dom}\left(S^{*}\right)
$$

and according to Second Representation Theorem the equalities

$$
\mathcal{D}[S]=\mathcal{D}\left[S_{F}\right]=\operatorname{Dom}\left(S_{F}^{1 / 2}\right), S[\varphi, \psi]=\left(S_{F}^{1 / 2} \varphi, S_{F}^{1 / 2} \psi\right), \varphi, \psi \in \operatorname{Dom}\left(S_{F}^{1 / 2}\right)
$$

hold.

Kreı̆n [37] established that any nonnegative, densely defined symmetric operator $S$ admits, so called, minimal nonnegative self-adjoint extension. This extension is called the Krel̆n-von Neumann extension $S_{K}$. The operator $S_{K}$ can be defined as follows [2,20]: $S_{K}=\left(\left(S^{-1}\right)_{F}\right)^{-1}$, where $S^{-1}$ denotes in this context the inverse nonnegative linear relation to the graph $S$. It was proved in [2] that

$$
\begin{gathered}
\mathcal{D}\left[S_{K}\right]=\operatorname{Dom}\left(S_{K}^{1 / 2}\right)=\left\{u \in H: \sup _{f \in \operatorname{Dom}(S)} \frac{|(u, S f)|^{2}}{(S f, f)}<\infty\right\}, \\
\sup _{f \in \operatorname{Dom}(S)} \frac{|(u, S f)|^{2}}{(S f, f)}=\left\|S_{K}^{1 / 2} u\right\|^{2}=S_{K}[u], u \in \mathcal{D}\left[S_{K}\right] .
\end{gathered}
$$

Krĕn proved that $\widetilde{S}$ is a nonnegative self-adjoint extension of $S$ if and only if

$$
S_{K} \leq \widetilde{S} \leq S_{F}
$$

in sense of the associated closed quadratic forms, i.e.,

$$
\begin{aligned}
& \mathcal{D}\left[S_{F}\right] \subseteq \mathcal{D}[\widetilde{S}] \subseteq \mathcal{D}\left[S_{K}\right], \\
& S_{F}[v]=\widetilde{S}[v], \quad v \in \mathcal{D}\left[S_{F}\right], \quad \widetilde{S}[u] \geq S_{K}[u], u \in \mathcal{D}[\widetilde{S}]
\end{aligned}
$$

Nonnegative self-adjoint extension $\widetilde{S}$ of $S$ is called extremal [4] if

$$
\inf \{(\widetilde{S}(u-x), u-x), x \in \operatorname{Dom}(S)\}=0 \text { for all } u \in \operatorname{Dom}(\widetilde{S}) \text {. }
$$


The Friedrichs and Kreı̆n-von Neumann extensions are extremal. The next theorem is established in [7].

Theorem 2.7 If $\widetilde{S}$ is a nonnegative self-adjoint extension of a nonnegative symmetric operator $S$, then the form

$$
(\widetilde{S} u, v)-S_{K}[u, v], u, v \in \operatorname{Dom}(\widetilde{S})
$$

is nonnegative and closable in the Hilbert space $\mathcal{D}\left[S_{K}\right]$. Moreover, the formulas

$$
\begin{aligned}
\mathcal{D}[\widetilde{S}] & =\operatorname{Dom}(\eta), \\
\widetilde{S}[u, v] & =S_{K}[u, v]+\eta[u, v], u, v \in \mathcal{D}[\widetilde{S}]
\end{aligned}
$$

give a one-to-one correspondence between all closed forms $\widetilde{S}[\cdot, \cdot]$ associated with nonnegative self-adjoint extensions $\widetilde{S}$ of $S$ and all nonnegative sesquilinear forms $\eta[\cdot, \cdot]$ closed in the Hilbert space $\mathcal{D}\left[S_{K}\right]$ and such that $\eta[\varphi]=0$ for all $\varphi \in \mathcal{D}[S]$. In addition, the closed form associated with extremal extensions are closed restrictions of the form $S_{K}[\cdot, \cdot]$ on the linear manifolds $\mathcal{M}$ such that

$$
\mathcal{D}[S] \subseteq \mathcal{M} \subseteq \mathcal{D}\left[S_{K}\right]
$$

Notice that investigations of all extremal extensions in more detail and their applications are presented in the paper [9].

Now we describe an approach proposed in [15-17] for parametrization of nonnegative self-adjoint extensions. Let $\mathfrak{N}_{F}$ be $(+)$-orthogonal complement of $\operatorname{Dom}(S)$ in Dom $\left(S_{F}\right)$, i.e.,

$$
\operatorname{Dom}\left(S_{F}\right)=\operatorname{Dom}(S) \oplus \mathfrak{N}_{F}
$$

Put $\mathfrak{M}_{F}=S_{F} \mathfrak{N}_{F}$. Then (see (2.19))

$$
\mathfrak{H}_{+}=\operatorname{Dom}(S) \oplus \mathfrak{N}_{F} \oplus \mathfrak{M}_{F}=\operatorname{Dom}\left(S_{F}\right) \oplus S_{F} \mathfrak{N}_{F},
$$

where decomposition is $(+)$-orthogonal decomposition. In addition

$$
S^{*} S_{F} e=-e, e \in \mathfrak{N}_{F}
$$

Let

$$
\mathfrak{N}_{0}=\operatorname{Ran}\left(S_{F}^{1 / 2}\right) \cap \mathfrak{N}_{F}
$$

Then $S$ has a unique nonnegative self-adjoint extension if and only if $\mathfrak{N}_{0}=\{0\}$ [15-17,37]. Suppose that $\mathfrak{N}_{0} \neq\{0\}$ and define the sesquilinear form on $\mathfrak{N}_{0}$

$$
w_{0}[e, g]=\left(S_{F}^{1 / 2} e, S_{F}^{1 / 2} g\right)+\left(\widehat{S}_{F}^{-1 / 2} e, \widehat{S}_{F}^{-1 / 2} g\right)=\left(\widehat{S}_{F}^{-1 / 2} e, \widehat{S}_{F}^{-1 / 2} g\right)_{+},
$$


where $\widehat{S}_{F}^{-1 / 2}$ denotes the Moore-Penrose inverse to $S_{F}^{1 / 2}$. This form is closed in $\mathfrak{H}_{+}$ and $w_{0}[e] \geq 2\|e\|^{2}$ for all $e \in \mathfrak{N}_{0}$. Let $\mathbf{W}_{0}$ be a (+)-nonnegative self-adjoint linear relation in $\mathfrak{N}_{F}$ associated with the closed form $w_{0}$. In view of $w_{0}[e]>0$ for all $e \neq 0 \in \mathfrak{N}_{0}$, the inverse 1.r. $\mathbf{W}_{0}^{-1}$ is densely defined in $\mathfrak{N}_{F}$ and therefore is the graph of a (+)-self-adjoint nonnegative operator. We denote this operator by $W_{0}^{-1}$. Clearly, Ker $W_{0}^{-1}=\mathbf{W}(0)=\mathfrak{N}_{F} \ominus \mathfrak{N}_{0}($ the $(+)$-orthogonal complement).

Theorem 2.8 The formulas

$$
\begin{aligned}
& \operatorname{Dom}(\widetilde{S})=\operatorname{Dom}(S) \oplus\left(I+S_{F} \widetilde{U}\right) \operatorname{Dom}(\widetilde{U}), \\
& \widetilde{S}\left(\varphi+h+S_{F} \widetilde{U} h\right)=S_{F}(\varphi+h)-\widetilde{U} h, \varphi \in \operatorname{Dom}(S), h \in \operatorname{Dom}(\widetilde{U}), \\
& \operatorname{Dom}\left(\widetilde{S}^{1 / 2}\right)=\operatorname{Dom}\left(S_{F}^{1 / 2}\right) \dot{+} S_{F} \operatorname{Ran}\left(\widetilde{U}^{1 / 2}\right), \\
& \left\|\widetilde{S}^{1 / 2}\left(f+S_{F} h\right)\right\|^{2}=\left\|S_{F}^{1 / 2} f-\widehat{S}_{F}^{-1 / 2} h\right\|^{2}+\widetilde{U}^{-1}[h]-w_{0}[h], \\
& f \in \operatorname{Dom}\left(S_{F}^{1 / 2}\right), h \in \operatorname{Ran}\left(\widetilde{U}^{1 / 2}\right)
\end{aligned}
$$

give a one-to-one correspondence between all non-negative self-adjoint extensions $\widetilde{S}$ of $S$ and their square roots and all (+)-self-adjoint operators $\widetilde{U}$ in $\mathfrak{N}_{F}$ satisfying the condition

$$
0 \leq \widetilde{U} \leq W_{0}^{-1}
$$

An extension $\widetilde{S}$ coincides with the Kre ̌n-von Neumann extensions $S_{K}$ iff $\widetilde{U}=W_{0}^{-1}$.

The extension $\widetilde{S}$ given by (2.27) is relatively prime with $S_{F}$ if and only if the operator $\widetilde{U}$ is invertible and transversal to $S_{F}$ iff $\widetilde{U}^{-1}$ is bounded.

Observe, that the condition (2.28) is equivalent to one of the following $[12,14]$ :

$$
\begin{aligned}
& \operatorname{Ran}(\widetilde{U}) \subset \mathfrak{N}_{0} \text { and }(\widetilde{U} f, f)_{+} \geq w_{0}[\widetilde{U} f] \text { for all } f \in \operatorname{Dom}(\widetilde{U}), \\
& \operatorname{Ran}(\widetilde{U}) \subset \mathfrak{N}_{0} \text { and }\left(\left(\widetilde{U} P_{\widetilde{U}}\right)^{-1} e, e\right)_{+} \geq w_{0}[e] \text { for all } e \in \operatorname{Ran}(\widetilde{U}),
\end{aligned}
$$

where $P_{\widetilde{U}}$ is the $(+)$-orthogonal projection in $\mathfrak{N}_{F}$ onto $\overline{\operatorname{Ran}}(\widetilde{U})$.

From Theorem 2.8 it follows that

$$
\begin{aligned}
\operatorname{Dom}\left(S_{K}^{1 / 2}\right) & =\operatorname{Dom}\left(S_{F}^{1 / 2}\right) \oplus S_{F} \mathfrak{N}_{0}, \\
\left\|S_{K}^{1 / 2}\left(f+S_{F} e\right)\right\|^{2} & =\left\|S_{F}^{1 / 2} f-\widehat{S}_{F}^{-1 / 2} e\right\|^{2}, f \in \operatorname{Dom}\left(S_{F}^{1 / 2}\right), e \in \mathfrak{N}_{0} .
\end{aligned}
$$

In addition from (2.29) it follows the relation

$$
\inf \left\{\left\|S_{K}^{1 / 2}(g-\psi)\right\|^{2}, \psi \in \operatorname{Dom}(S)\right\}=0 \text { for all } g \in \operatorname{Dom}\left(S_{K}^{1 / 2}\right)
$$


2.8 Quasi-Self-Adjoint m-Accretive and m-Sectorial Extensions of Nonnegative Symmetric Operators via Fractional-Linear Transformations

Let $A$ be a nondensely defined Hermitian contraction in the Hilbert space $\mathfrak{H}$ with the domain $\operatorname{Dom}(A)=: \mathfrak{H}_{0}$ and let $\mathcal{N}:=\mathfrak{H} \ominus \operatorname{Dom}(A)$. Let $P_{0}$ and $P_{\mathcal{N}}$ be the orthogonal projections in $\mathfrak{H}$ onto $\mathfrak{H}_{0}$ and $\mathcal{N}$, respectively. Then the operator $A_{0}=P_{0} A$ is contractive and self-adjoint in the subspace $\mathfrak{H}_{0}$. Let $D_{A_{0}}=\left(I-A_{0}^{2}\right)^{1 / 2}$ be the defect operator determined by $A_{0}$. The operator $A_{21}=P_{\mathcal{N}} A$ is also contractive. Moreover, it follows from $A^{*} A \leq I$ that $A_{21}^{*} A_{21} \leq D_{A_{0}}^{2}$. Therefore, the identity

$$
K_{0} D_{A_{0}} f=P_{\mathcal{N}} A f, f \in \operatorname{Dom}(A),
$$

defines a contractive operator $K_{0}$ from $\mathfrak{D}_{A_{0}}:=\overline{\operatorname{Ran}}\left(D_{A_{0}}\right)$ into $\mathcal{N}$, cf. [27,30]. This gives the following decomposition for the Hermitian contraction $A$

$$
A=A_{0}+K_{0} D_{A_{0}}=\left(\begin{array}{c}
A_{0} \\
K_{0} D_{A_{0}}
\end{array}\right) .
$$

Let the Hermitian contraction $A$ in $\mathfrak{H}$ be defined on the subspace $\mathfrak{H}_{0}=\operatorname{Dom}(A)$. A linear operator $T$ is called quasi-self-adjoint contractive extension of $A$ ( $q s c$-extension of $A)[11,12,14]$ if

$$
\operatorname{Dom}(T)=\mathfrak{H}, T \supset A, T^{*} \supset A,\|T\| \leq 1 .
$$

It was established by Kreı̆n [37] that the set of all contractive extensions of $A$ forms an operator interval $\left[A_{\mu}, A_{M}\right]$, where the endpoints posses the properties

$$
\begin{aligned}
& \inf \left\{\left(\left(I+A_{\mu}\right)(h-\phi), h-\phi\right), \phi \in \mathfrak{H}_{0}\right\}=0, \\
& \inf \left\{\left(\left(I-A_{M}\right)(h-\phi), h-\phi\right), \phi \in \mathfrak{H}_{0}\right\}=0
\end{aligned}
$$

for all $h \in \mathfrak{H}$. These equalities are equivalent to [37]

$$
\operatorname{Ran}\left(\left(I+A_{\mu}\right)^{1 / 2}\right) \cap \mathcal{N}=\{0\}, \quad \operatorname{Ran}\left(\left(I-A_{M}\right)^{1 / 2}\right) \cap \mathcal{N}=\{0\}
$$

Moreover, it is proved in [37] that if $S$ is a densely defined closed symmetric and nonnegative operator in $H$ and if $A=(I-S)(I+S)^{-1}$, then

$$
S_{F}=\left(I-A_{\mu}\right)\left(I+A_{\mu}\right)^{-1}
$$

and the operator

$$
S_{M}=\left(I-A_{M}\right)\left(I+A_{M}\right)^{-1}
$$

is the minimal nonnegative self-adjoint extension of $S$. Thus $S_{M}$ coincides with the Kreĭn-von-Neumann extension $S_{K}$ of $S$. 

ball

In $[11,12]$ it is established that the set of all $q s c$-extensions of $A$ forms the operator

$$
\mathfrak{B}\left(\frac{A_{\mu}+A_{M}}{2}, \frac{A_{M}-A_{\mu}}{2}\right)
$$

with the center $\left(A_{\mu}+A_{M}\right) / 2$ and equal left and right radii $R_{l}=R_{r}=\left(A_{M}-\right.$ $\left.A_{\mu}\right)^{1 / 2} / \sqrt{2}$, i.e., there is a one-to-one correspondence between all $q s c$-extensions $T$ of $A$ and all contractions $X$ in $\mathcal{N}_{0}:=\overline{\operatorname{Ran}}\left(A_{M}-A_{\mu}\right)$ given by the relation

$$
T=\frac{A_{\mu}+A_{M}}{2}+\left(\frac{A_{M}-A_{\mu}}{2}\right)^{1 / 2} X\left(\frac{A_{M}-A_{\mu}}{2}\right)^{1 / 2}
$$

As is shown in [13] the qsc-extension $T$ belongs to the class $C_{\mathfrak{H}}(\alpha)$ if and only if the contraction $X$ in (2.33) belong to the class $C_{\mathcal{N}_{0}}(\alpha)$.

Decompose $A$ according to $\mathfrak{H}=\mathfrak{H}_{0} \oplus \mathcal{N}$ as in (2.31). Let $T$ be a $q s c$-extension of $A$ and decompose $T=\left(T_{i j}\right)$ also with respect to $\mathfrak{H}=\mathfrak{H}_{0} \oplus \mathcal{N}$. Then clearly $T_{11}=A_{0}, T_{12}^{*}=T_{21}=K_{0} D_{A_{0}}$. The next result gives a parametrization of all $q s c$ extensions of $A$ and some of its subclasses by means of block formulas, cf. [19,22,49], and $[11,14]$.

Theorem 2.9 Let $A$ be a Hermitian contraction in $\mathfrak{H}=\mathfrak{H}_{0} \oplus \mathcal{N}$ with $\operatorname{Dom}(A)=\mathfrak{H}_{0}$ and decompose A as in (2.31). Then:

(i) the formula

$$
T=\left(\begin{array}{cc}
A_{0} & D_{A_{0}} K_{0}^{*} \\
K_{0} D_{A_{0}} & -K_{0} A_{0} K_{0}^{*}+D_{K_{0}^{*}} X D_{K_{0}^{*}}
\end{array}\right): \begin{array}{cc}
\mathfrak{H}_{0} & \mathfrak{H}_{0} \\
\underset{\mathcal{N}}{\oplus} & \rightarrow \\
\mathcal{N}
\end{array}
$$

gives a one-to-one correspondence between all qsc-extensions $T$ of the Hermitian contraction $A=A_{0}+K_{0} D_{A_{0}}$ and all contractions $X$ in the subspace $\mathfrak{D}_{K_{0}^{*}}:=\overline{\operatorname{Ran}\left(D_{\left.K_{0}^{*}\right)}\right.} \subset \mathcal{N}$;

(ii) $T$ in (2.34) belongs to the class $C_{\mathfrak{H}}(\alpha)$ if and only if $X$ belongs to the class $\left.C_{\mathfrak{D}_{K_{0}^{*}}}(\alpha), \alpha \in\right] 0, \pi / 2[$;

(iii) $T$ is a self-adjoint extension of $A$ if and only if $X$ in (2.34) is a self-adjoint contraction in $\mathfrak{D}_{K_{0}^{*}}$.

From (2.34) it follows that

$$
\begin{aligned}
A_{\mu} & =\left(\begin{array}{cc}
A_{0} & D_{A_{0}} K_{0}^{*} \\
K_{0} D_{A_{0}} & -K_{0} A_{0} K_{0}^{*}-D_{K_{0}^{*}}^{2}
\end{array}\right), \\
A_{M} & =\left(\begin{array}{cc}
A_{0} & D_{A_{0}} K_{0}^{*} \\
K_{0} D_{A_{0}} & -K_{0} A_{0} K_{0}^{*}+D_{K_{0}^{*}}^{2}
\end{array}\right)
\end{aligned}
$$


with $X=-I\left\lceil\mathfrak{D}_{K_{0}^{*}}\right.$ and $X=I\left\lceil\mathfrak{D}_{K_{0}^{*}}\right.$, respectively. From the formulas (2.35) it is seen that

$$
\frac{A_{\mu}+A_{M}}{2}=\left(\begin{array}{cc}
A_{0} & D_{A_{0}} K_{0}^{*} \\
K_{0} D_{A_{0}} & -K_{0} A_{0} K_{0}^{*}
\end{array}\right), \quad \frac{A_{M}-A_{\mu}}{2}=\left(\begin{array}{cc}
0 & 0 \\
0 & D_{K_{0}^{*}}^{2}
\end{array}\right) .
$$

It is easy to see from (2.34) and (2.35) that if $T$ is a $q s c$-extension of $A$ such that $T_{R}=\left(T+T^{*}\right) / 2=A_{\mu}\left(A_{M}\right)$, then in fact $T=A_{\mu}\left(A_{M}\right)$. Namely, $X=X_{R}+i X_{I}$ satisfies

$$
\left\{\begin{array}{l}
0 \leq X^{*} X=X_{R}^{2}+i\left(X_{R} X_{I}-X_{I} X_{R}\right)+X_{I}^{2} \leq I \\
0 \leq X X^{*}=X_{R}^{2}-i\left(X_{R} X_{I}-X_{I} X_{R}\right)+X_{I}^{2} \leq I
\end{array}\right.
$$

so that $0 \leq X_{R}^{2}+X_{I}^{2} \leq I$ and here clearly $X_{R}^{2}=I$ implies $X_{I}=0$.

Remark 2.10 Block formulas for describing all contractive extensions of a dual pair, in particular qsc-extensions of a Hermitian contraction, appear in $[19,22,49]$.

\section{Parametrization of all Quasi-Self-Adjoint m-Accretive Extensions}

In this section we develop a method described in Sect. 2.7 (see Theorem 2.8) to the problem of $m$-accretive quasi-self-adjoint extensions. We need the followings results established in [6].

Theorem 3.1 Let $S$ be a nonnegative symmetric operator and let $\widetilde{S}$ be a $m$-accretive extension of $S$. The following conditions are equivalent:

(i) $\widetilde{S} \subset S^{*}$;

(ii) $\operatorname{Dom}(\tilde{S}) \subset \mathcal{D}\left[S_{K}\right]$ and

$$
\operatorname{Re}(\widetilde{S} f, f) \geq S_{K}[f]=\left\|S_{K}^{1 / 2} f\right\|^{2} \text { for all } f \in \operatorname{Dom}(\widetilde{S})
$$

(iii)

$$
|(S g, f)|^{2} \leq(S g, g) \operatorname{Re}(\widetilde{S} f, f) \text { for all } f \in \operatorname{Dom}(\widetilde{S}), g \in \operatorname{Dom}(S)
$$

The extension $\widetilde{S}$ is quasi-self-adjoint and $m-\alpha$-sectorial if and only if the sesquilinear form

$$
w[f, h]=(\widetilde{S} f, h)-S_{K}[f, h], f, h \in \operatorname{Dom}(\widetilde{S})
$$

is $\alpha$-sectorial.

Proof If $\widetilde{S}$ is an accretive extension of $S$ then for all $g \in \operatorname{Dom}(S)$, for all $f \in \operatorname{Dom}(\widetilde{S})$, and for all $t \in \mathbb{R}$ it follows

$$
0 \leq \operatorname{Re}(\widetilde{S}(t g+f), t g+f)=t^{2}(S g, g)+t(\operatorname{Re}(S g, f)+\operatorname{Re}(\widetilde{S} f, g))+\operatorname{Re}(\widetilde{S} f, f)
$$


If in addition $\widetilde{S} \subset S^{*}$, then $\operatorname{Dom}(\widetilde{S}) \subset \operatorname{Dom}\left(S^{*}\right)$ and $(S g, f)=(g, \widetilde{S} f)$. Hence

$$
t^{2}(S g, g)+2 t \operatorname{Re}(S g, f)+\operatorname{Re}(\widetilde{S} f, f) \geq 0
$$

for all $t \in \mathbb{R}$. Now we get

$$
|\operatorname{Re}(S g, f)|^{2} \leq(S g, g) \operatorname{Re}(\widetilde{S} f, f)
$$

and therefore

$$
|(S g, f)|^{2} \leq(S g, g) \operatorname{Re}(\widetilde{S} f, f)
$$

for all $g \in \operatorname{Dom}(S)$ and all $f \in \operatorname{Dom}(\widetilde{S})$, i.e., (i) $\Rightarrow$ (iii). The equivalence (iii) $\Longleftrightarrow$ (ii) follows from (2.23).

Let us show that (iii) implies (i). Let $A=(I-S)(I+S)^{-1}$ and $\widetilde{A}=(I-\widetilde{S})(I+\widetilde{S})^{-1}$. Then $A$ is Hermitian contraction defined on $\operatorname{Dom}(A)=(I+S) \operatorname{Dom}(S)$ and $\widetilde{A}$ is contractive extension of $A$ defined on $\mathfrak{H}$. Then the inequality in (iii) can be rewritten as follows

$$
|((I-A) \varphi,(I+\widetilde{A}) h)|^{2} \leq((I-A) \varphi,(I+A) \varphi) \operatorname{Re}((I-\widetilde{A}) h,(I+\widetilde{A}) h)
$$

for all $\varphi \in \operatorname{Dom}(A)$ and all $h \in \mathfrak{H}$. Using the relation $A \varphi=\widetilde{A} \varphi$ and simplifying we obtain

$\left|\left(D_{\widetilde{A}}^{2} \varphi-2 i \widetilde{A}_{I} \varphi, h\right)\right|^{2} \leq\left\|D_{\widetilde{A}} \varphi\right\|^{2}\left\|D_{\widetilde{A}} h\right\|^{2}$ for all $\varphi \in \operatorname{Dom}(A)$ and all $h \in \mathfrak{H}$.

From (2.2) we obtain that

$$
D_{\widetilde{A}}^{2} \varphi-2 i \widetilde{A}_{I} \varphi \in \operatorname{Ran}\left(D_{\widetilde{A}}\right) \text { for all } \varphi \in \operatorname{Dom}(A)
$$

and

$$
\left\|\widehat{D}_{\widetilde{A}}^{-1}\left(D_{\widetilde{A}}^{2} \varphi-2 i \widetilde{A}_{I} \varphi\right)\right\|^{2} \leq\left\|D_{\widetilde{A}} \varphi\right\|^{2}, \varphi \in \operatorname{Dom}(A) .
$$

Since $D_{\widetilde{A}}^{2} \varphi \in \operatorname{Ran}\left(D_{\widetilde{A}}\right)$, we get $\widetilde{A}_{I} \varphi \in \operatorname{Ran}(\widetilde{A})$ and

$$
\left\|D_{\widetilde{A}} \varphi-2 i \widehat{D}_{\widetilde{A}}^{-1} \widetilde{A}_{I} \varphi\right\|^{2} \leq\left\|D_{\widetilde{A}} \varphi\right\|^{2}, \varphi \in \operatorname{Dom}(A)
$$

Hence

$$
\left\|D_{\widetilde{A}} \varphi\right\|^{2}+4\left\|\widehat{D}_{\widetilde{A}}^{-1} \widetilde{A}_{I} \varphi\right\|^{2} \leq\left\|D_{\widetilde{A}} \varphi\right\|^{2}
$$

It follows that $\widetilde{A}_{I} \varphi=0$ for all $\varphi \in \operatorname{Dom}(A)$. This means that $\widetilde{A}^{*} \supset A$, i.e., $\widetilde{A}$ is a $q s c$-extension of $A$. Therefore, $\widetilde{S}$ is a quasi-self-adjoint extension of $S$. 
Suppose that the sesquilinear form $\omega$ given by (3.1) is $\alpha$-sectorial. Then $\operatorname{Re}(\widetilde{S} f, f) \geq S_{K}[f]$ for all $f \in \operatorname{Dom}(\widetilde{S})$. Therefore $\widetilde{S}$ is m-accretive and quasiself-adjoint extension of $S$. On the other hand for all $f \in \operatorname{Dom}(\widetilde{S})$ we have

$$
\tan \alpha \operatorname{Re}(\widetilde{S} f, f) \pm \operatorname{Im}(\widetilde{S} f, f)=\tan \alpha \operatorname{Re} \omega[f] \pm \operatorname{Im} \omega[f]+S_{K}[f] \geq 0
$$

Hence $\widetilde{S}$ is m- $\alpha$-sectorial extension of $S$.

Conversely, let $\widetilde{S}$ is quasi-self-adjoint and $\mathrm{m}-\alpha$-sectorial extension of $S$. Hence $\operatorname{Dom}(\widetilde{S}) \subset \mathcal{D}\left[S_{K}\right]$. Since for each $\varphi \in \operatorname{Dom}(S) \subset \operatorname{Dom}\left(S_{K}\right)$ and all $f \in \mathcal{D}\left[S_{K}\right]$ one has $S_{K}[f, \varphi]=(f, S \varphi)$ and $S_{K}[\varphi, f]=(S \varphi, f)$, we get

$$
\omega[f-\varphi]=\omega[f]
$$

for all $f \in \operatorname{Dom}(\widetilde{S})$ and all $\varphi \in \operatorname{Dom}(S)$. Because

$$
\inf _{\varphi \in \operatorname{Dom}(S)} S_{K}[f-\varphi]=0
$$

for all $f \in \mathcal{D}\left[S_{K}\right]$, for given $f \in \mathcal{D}\left[S_{K}\right]$ and for every $\varepsilon>0$ one can find $\varphi_{0} \in$ $\operatorname{Dom}(S)$ such that

$$
S_{K}\left[f-\varphi_{0}\right]<\varepsilon
$$

It follows that

$$
\begin{aligned}
\tan \alpha \operatorname{Re} \omega[f] \pm \operatorname{Im} \omega[f]= & \tan \alpha \operatorname{Re} \omega\left[f-\varphi_{0}\right] \pm \operatorname{Im} \omega\left[f-\varphi_{0}\right] \\
= & \tan \alpha \operatorname{Re}\left(\widetilde{S}\left(f-\varphi_{0}\right), f-\varphi_{0}\right) \\
& \pm \operatorname{Im}\left(\widetilde{S}\left(f-\varphi_{0}\right), f-\varphi_{0}\right)-S_{K}\left[f-\varphi_{0}\right]>-\varepsilon
\end{aligned}
$$

Since $\varepsilon$ is an arbitrary positive number, the form $\omega$ is $\alpha$-sectorial.

Remark 3.2 In addition to the statements in Theorem 2.7 from results obtained in [7] follows that the relations

$$
\mathcal{D}[\widetilde{S}]=\operatorname{Dom}(\eta), \widetilde{S}[u, v]=S_{K}[u, v]+\eta[u, v]
$$

establish a one-to-one correspondence between all closed forms associated with quasiself-adjoint m- $\alpha$-sectorial extensions of nonnegative $S$ and all sesquilinear $\alpha$-sectorial forms $\eta$ closed in the Hilbert space $\mathcal{D}\left[S_{K}\right]$ and such that $\eta[\varphi]=0$ for all $\varphi \in \mathcal{D}[S]$.

Corollary 3.3 [50] If $S_{F}=S_{K}$ and if $\widetilde{S}$ is m-accretive quasi-self-adjoint extension of $S$, then $\widetilde{S}=S_{F}$.

Proof Since $S_{F}$ coincides with $S_{K}$, from Theorem 3.1 it follows that $\operatorname{Dom}(\widetilde{S}) \subset$ $\mathcal{D}\left[S_{F}\right]$. But $\operatorname{Dom}(\widetilde{S}) \subset \operatorname{Dom}\left(S^{*}\right)$ and $\operatorname{Dom}\left(S^{*}\right) \cap \mathcal{D}\left[S_{F}\right]=\operatorname{Dom}\left(S_{F}\right)$. Hence, $\widetilde{S}=S_{F}$. 
Next theorem gives a parametrization of all quasi-self-adjoint $m$-accretive and $m$-sectorial extensions of $S$.

Theorem 3.4 The formulas

$$
\begin{aligned}
\operatorname{Dom}(\widetilde{S}) & =\operatorname{Dom}(S) \oplus\left(I+S_{F} \widetilde{U}\right) \operatorname{Dom}(\widetilde{U}), \\
\widetilde{S}\left(\varphi+h+S_{F} \widetilde{U} h\right) & =S_{F}(\varphi+h)-\widetilde{U} h, \varphi \in \operatorname{Dom}(S), h \in \operatorname{Dom}(\widetilde{U})
\end{aligned}
$$

give a one-to-one correspondence between all m-accretive quasi-self-adjoint extensions $\widetilde{S}$ of $S$ and all (+)-m-accretive operators $\widetilde{U}$ in $\mathfrak{N}_{F}$ satisfying the condition

$$
\operatorname{Ran}(\tilde{U}) \subset \mathfrak{N}_{0} \text { and } \operatorname{Re}(\widetilde{U} e, e)_{+} \geq w_{0}[\tilde{U} e] \text { for all } e \in \operatorname{Dom}(\widetilde{U})
$$

The extension $\widetilde{S}$ in (3.2) is $m$ - $\alpha$-sectorial if and only if

$\widetilde{U}$ in $\mathfrak{N}_{F}$ is $(+)-m$-accretive, $\operatorname{Ran}(\widetilde{U}) \subset \mathfrak{N}_{0}$,

the sesquilinear form $\tau_{\widetilde{U}}[e, h]:=(\widetilde{U} e, h)_{+}-w_{0}[\widetilde{U} e, \widetilde{U} h], e, h \in \operatorname{Dom}(\widetilde{U})$,

is $\alpha$-sectorial.

If this is the case, then associated with $\widetilde{S}$ closed form $\widetilde{S}[\cdot, \cdot]$ is given by

$$
\begin{aligned}
\mathcal{D}[\widetilde{S}]= & \mathcal{D}[S] \dot{+} S_{F} \mathcal{D}\left[\widetilde{U}^{-1}\right], \\
\widetilde{S}\left[\varphi_{1}+S_{F} h_{1}, \varphi_{2}+S_{F} h_{2}\right]= & \left(S_{F}^{1 / 2} \varphi_{1}-\widehat{S}_{F}^{-1 / 2} h_{1}, S_{F}^{1 / 2} \varphi_{2}-\widehat{S}_{F}^{-1 / 2} h_{2}\right) \\
& +\widetilde{U}^{-1}\left[h_{1}, h_{2}\right]-w_{0}\left[h_{1}, h_{2}\right], \\
& \varphi_{1}, \varphi_{2} \in \mathcal{D}[S], h_{1}, h_{2} \in \mathcal{D}\left[\widetilde{U}^{-1}\right] .
\end{aligned}
$$

The extension $\widetilde{S}$ in (3.2) is relatively prime with $S_{F}$ iff the operator $\widetilde{U}$ is invertible, $\widetilde{S}$ is transversal to $S_{F}$ iff $\widetilde{U}^{-1}$ is bounded.

Proof Suppose that $\widetilde{S}$ is accretive quasi-self-adjoint extension of $S$. Then $\operatorname{Dom}(\widetilde{S}) \cap$ $\mathfrak{M}_{F}=\{0\}$. In fact if $e \in \operatorname{Dom}(\widetilde{S}) \cap \mathfrak{M}_{F}$, then $e=S_{F} g, g \in \mathfrak{N}_{F}$ and $\widetilde{S} e=S^{*} S_{F} g=$ $-g, \operatorname{Re}(\widetilde{S} e, e)=-\left(g, S_{F} g\right) \leq 0$. Since $S_{F}$ is nonnegative self-adjoint operator, it follows that $e=S_{F} g=0$.

This implies that the domain $\operatorname{Dom}(\widetilde{S})$ can be represented as follows

$$
\operatorname{Dom}(\widetilde{S})=\operatorname{Dom}(S) \oplus\left(I+S_{F} \widetilde{U}\right) \operatorname{Dom}(\widetilde{U}),
$$

where $\widetilde{U}$ is a linear operator in $\mathfrak{N}_{F}$ with some domain $\operatorname{Dom}(\widetilde{U})$.

Let us show that $\widetilde{U}$ is a (+)-accretive operator in $\mathfrak{N}_{F}$. Consider an arbitrary vector $f \in \operatorname{Dom}(\widetilde{S})$ of the form $f=h+S_{F} \widetilde{U} h, h \in \operatorname{Dom}(\widetilde{U})$. Then $\widetilde{S} f=S^{*} f=$ 
$S_{F} h-\widetilde{U} h$ and

$$
\begin{aligned}
(\widetilde{S} f, f)= & \left(S_{F} h-\widetilde{U} h, h+S_{F} \widetilde{U} h\right) \\
= & \left(S_{F} h, h\right)-\left(\widetilde{U} h, S_{F} \widetilde{U} h\right)+\left(S_{F} h, S_{F} \widetilde{U} h\right)-(\widetilde{U} h, h) \\
= & \left(S_{F} h, h\right)-\left(\widetilde{U} h, S_{F} \widetilde{U} h\right)+\left(S_{F} h, S_{F} \widetilde{U} h\right)+(h, \widetilde{U} h) \\
& -2 \operatorname{Re}(\widetilde{U} h, h) \\
= & \left(S_{F} h, h\right)-\left(\widetilde{U} h, S_{F} \widetilde{U} h\right)+(h, \widetilde{U} h)_{+}-2 \operatorname{Re}(\widetilde{U} h, h) .
\end{aligned}
$$

Since $\widetilde{S}$ is an accretive quasi-self-adjoint extension of $S$, by Theorem 3.1 every vector $f \in \operatorname{Dom}(\widetilde{S})$ belongs to $\operatorname{Dom}\left(S_{K}^{1 / 2}\right)$ and the inequality $\operatorname{Re}(\widetilde{S} f, f) \geq$ $\left\|S_{K}^{1 / 2} f\right\|^{2}$ holds. From (2.29) it follows that $\operatorname{Ran}(\widetilde{U}) \subset \mathfrak{N}_{0}$ and for $f=h+$ $S_{F} \widetilde{U} h, h \in \operatorname{Dom}(\widetilde{U})$ holds

$\left\|S_{F}^{1 / 2} h-\widehat{S}_{F}^{-1 / 2} \widetilde{U} h\right\|^{2} \leq\left(S_{F} h, h\right)-\left(\widetilde{U} h, S_{F} \widetilde{U} h\right)+\operatorname{Re}(h, \widetilde{U} h)_{+}-2 \operatorname{Re}(\widetilde{U} h, h)$.

Since

$$
\left\|S_{F}^{1 / 2} h-\widehat{S}_{F}^{-1 / 2} \widetilde{U} h\right\|^{2}=\left(S_{F} h, h\right)+\left\|S_{F}^{-1 / 2} \widetilde{U} h\right\|^{2}-2 \operatorname{Re}(\widetilde{U} h, h),
$$

we get

$$
\left\|\widehat{S}_{F}^{-1 / 2} \widetilde{U} h\right\|^{2}+\left\|S_{F}^{1 / 2} \widetilde{U} h\right\|^{2} \leq \operatorname{Re}(h, \widetilde{U} h)_{+} .
$$

By (2.26) we get

$$
w_{0}[\widetilde{U} h] \leq \operatorname{Re}(\widetilde{U} h, h)_{+} \text {for all } h \in \operatorname{Dom}(\widetilde{U})
$$

This inequality yields that the operator $\widetilde{U}$ is $(+)$-accretive.

Suppose now that $\widetilde{S}$ is m-accretive operator. Then its adjoint $\widetilde{S}^{*}$ is also m-accretive and is a quasi-self-adjoint extension of $S$. In this case the operator $\widetilde{U}$ is (+)-closed and has dense domain. Indeed, if the vector $e \in \mathfrak{N}_{F}$ is (+)-orthogonal to $\operatorname{Dom}(\widetilde{U})$, i.e., $(e, h)_{+}=0$ for all $h \in \operatorname{Dom}(\widetilde{U})$ then by definition of the inner product $(\cdot, \cdot)_{+}$ we have

$$
\left(S_{F} e, S_{F} h\right)+(e, h)=0, h \in \operatorname{Dom}(\widetilde{U}) .
$$

Using (+)-orthogonality $S_{F} \mathfrak{N}_{F}$ to $\operatorname{Dom}(S) \dot{+} \mathfrak{N}_{F}$, one obtains that for every $\varphi \in \operatorname{Dom}(S)$

$$
\left(-e, \varphi+h+S_{F} \widetilde{U} h\right)=\left(S_{F} e, S \varphi+S_{F} h-\widetilde{U} h\right)
$$

The latter means that the vector $S_{F} e$ belongs to $\operatorname{Dom}\left(\widetilde{S}^{*}\right)$. It is shown above that $e=0$. Thus, if $\widetilde{S}$ is m-accretive quasi-self-adjoint extension of $S$, then the corresponding operator $\widetilde{U}$ is (+)-closed, densely defined in $\mathfrak{N}_{F},(+)$-accretive and satisfies 
condition (3.6). Moreover, for the adjoint $\widetilde{S}^{*}$ holds the decomposition

$$
\operatorname{Dom}\left(\widetilde{S}^{*}\right)=\operatorname{Dom}(S) \oplus\left(I+S_{F} \widetilde{U}^{*}\right) \operatorname{Dom}\left(\widetilde{U}^{*}\right),
$$

where $\widetilde{U}^{*}$ is the $(+)$-adjoint to $\widetilde{U}$ in $\mathfrak{N}_{F}$. Since $\widetilde{S}^{*}$ is accretive, the operator $\widetilde{U}^{*}$ is $\left(+\right.$ )-accretive (and also satisfies the condition (3.6) with replacement $\widetilde{U}$ by $\widetilde{U}^{*}$ ). Because $\widetilde{U}$ and $\widetilde{U}^{*}$ are both (+)-accretive operators, the operator $\widetilde{U}$ (as well as $\widetilde{U}^{*}$ ) is (+)-m-accretive in the subspace $\mathfrak{N}_{F}$ and satisfies (3.6).

Conversely, suppose that $\widetilde{U}$ in $\mathfrak{N}_{F}$ satisfies (3.3). Let the operator $\widetilde{S}$ be given by (3.2). Then $\widetilde{S}$ is closed quasi-self-adjoint extension of $S$ and one can verify that for the vector $f=h+S_{F} \widetilde{U} h$ the condition $\operatorname{Re}(\widetilde{S} f, f) \geq\left\|S_{K} f\right\|^{2}$ holds. Therefore, from (2.23) it follows that

$$
|(S \varphi, f)|^{2} \leq(S \varphi, \varphi) \operatorname{Re}(\widetilde{S} f, f)
$$

for all $\varphi \in \operatorname{Dom}(S)$. The last inequality yields $|(S \varphi, f)| \leq(S \varphi, \varphi)+\operatorname{Re}(\widetilde{S} f, f)$. Hence,

$$
\begin{aligned}
& \operatorname{Re}(\widetilde{S}(\varphi+f), \varphi+f)=(S \varphi, \varphi)+\operatorname{Re}(\widetilde{S} f, f)+2 \operatorname{Re}(\widetilde{S} \varphi, f) \\
& \geq(S \varphi, \varphi)+\operatorname{Re}(\widetilde{S} f, f)-(S \varphi, \varphi)-\operatorname{Re}(\widetilde{S} f, f)=0 .
\end{aligned}
$$

Thus, the operator $\widetilde{S}$ is accretive. Consider the pair $\langle S, \widetilde{S}\rangle$. Because $(S \varphi, g)=(\varphi, \widetilde{S} g)$ for all $\varphi \in \operatorname{Dom}(S)$ and all $g \in \operatorname{Dom}(\widetilde{S})$ and $\widetilde{S}$ is closed accretive operator, there exists [48] a m-accretive operator $\widetilde{S}^{\prime}$ such that $\widetilde{S}^{\prime} \supset \widetilde{S}$ and $\widetilde{S}^{\prime} \supset S$. Therefore,

$$
S \subset \widetilde{S} \subset \widetilde{S}^{\prime} \subset S^{*}
$$

This means that $\widetilde{S}^{\prime}$ is quasi-self-adjoint m-accretive extension of $S$. Since $\widetilde{S}^{\prime}$ extends $\widetilde{S}$, the corresponding operator $\widetilde{U}^{\prime}$ in the representation

$$
\operatorname{Dom}\left(\widetilde{S}^{\prime}\right)=\operatorname{Dom}(S) \oplus\left(I+S_{F} \widetilde{U}^{\prime}\right) \operatorname{Dom}\left(\widetilde{U}^{\prime}\right),
$$

is (+)-accretive extension in $\mathfrak{N}_{F}$ of the operator $\widetilde{U}$. Because $\widetilde{U}$ is $\mathrm{m}$-accretive, we get the equality $\widetilde{U}^{\prime}=\widetilde{U}$ and therefore $\widetilde{S}^{\prime}=\widetilde{S}$, i.e. $\widetilde{S}$ already is m-accretive extension of $S$.

Since null-spaces of a m-accretive operator and its adjoint coincide, the condition (3.6) is equivalent to the condition (3.3).

Now suppose that quasi-self-adjoint and m-accretive extension $\widetilde{S}$ of $S$ is given by (3.2). Let $\varphi \in \operatorname{Dom}(S), h \in \operatorname{Dom}(\widetilde{U})$, and let $g=\varphi+h+S_{F} \widetilde{U} h$. Then taking into account that $(\widetilde{U} h, \varphi)_{+}=0$, and therefore $(\widetilde{U} h, \varphi)=-\left(S_{F} \widetilde{U} h, S_{F} \varphi\right)$, we get

$$
\begin{aligned}
(\widetilde{S} g, g)= & \left(S_{F}(\varphi+h)-\widetilde{U} h, \varphi+h+S_{F} \widetilde{U} h\right)=\left(S_{F}(\varphi+h), \varphi+h\right) \\
& +\left(S_{F}(\varphi+h), S_{F} \widetilde{U} h\right)-(\widetilde{U} h, \varphi+h)-\left(\widetilde{U} h, S_{F} \widetilde{U} h\right) \\
= & \left(S_{F}(\varphi+h), \varphi+h\right)+2 \operatorname{Re}\left(S_{F} \widetilde{U} h, S_{F} \varphi\right)+\left(\widetilde{U} h, S_{F} \widetilde{U} h\right)-2 \operatorname{Re}(\widetilde{U} h, h) \\
& +(h, \widetilde{U} h)_{+} .
\end{aligned}
$$


From (2.29) it follows

$$
\begin{aligned}
\left\|S_{K}^{1 / 2} g\right\|^{2} & =\left\|S_{F}^{1 / 2}(\varphi+h)-\widehat{S}_{F}^{-1 / 2} \widetilde{U} h\right\|^{2} \\
& =\left(S_{F}(\varphi+h), \varphi+h\right)+\left\|\widehat{S}_{F}^{-1 / 2} \widetilde{U} h\right\|^{2}-2 \operatorname{Re}(\varphi+h, \widetilde{U} h) \\
& =\left(S_{F}(\varphi+h), \varphi+h\right)+\left\|\widehat{S}_{F}^{-1 / 2} \widetilde{U} h\right\|^{2}-2 \operatorname{Re}(h, \widetilde{U} h)+2 \operatorname{Re}\left(S_{F} \varphi, S_{F} \widetilde{U} h\right) .
\end{aligned}
$$

Since $\left\|S_{F}^{1 / 2} \widetilde{U} h\right\|^{2}+\left\|\widehat{S}_{F}^{-1 / 2} \widetilde{U} h\right\|^{2}=w_{0}[\widetilde{U} h]$, we obtain

$$
(\widetilde{S} g, g)-\left\|S_{K}^{1 / 2} g\right\|^{2}=(h, \widetilde{U} h)_{+}-w_{0}[\widetilde{U} h] .
$$

According Theorem 3.1 the operator $\widetilde{S}$ is $\alpha$-sectorial if and only if the quadratic form

$$
(\widetilde{S} g, g)-\left\|S_{K}^{1 / 2} g\right\|^{2}, g \in \operatorname{Dom}(\widetilde{S})
$$

is $\alpha$-sectorial. Now from (3.7) it follows that the operator $\widetilde{S}$ is $\alpha$-sectorial if and only if the form

$$
\tau_{\widetilde{U}}[e, h]=(e, \widetilde{U} h)_{+}-w_{0}[\widetilde{U} e, \widetilde{U} h], e, h \in \operatorname{Dom}(\widetilde{U})
$$

is $\alpha$-sectorial.

Observe that from conditions (3.4) it follows that the operator $\widetilde{U}$ and the inverse linear relation $\widetilde{U}^{-1}$ are $\alpha$-sectorial. Hence, the form $\left(\widetilde{U}^{-1} e, h\right)_{+}$has the closure $\widetilde{U}^{-1}[\cdot, \cdot]$ in $\mathfrak{N}_{F}$. Moreover, $\mathcal{D}\left[\widetilde{U}^{-1}\right] \subseteq \mathfrak{N}_{0}=\operatorname{Dom}\left(w_{0}\right)$ and the sesquilinear form

$$
\nu_{\widetilde{U}}[e, h]:=\widetilde{U}^{-1}[e, h]-w_{0}[e, h], e, h \in \mathcal{D}\left[\widetilde{U}^{-1}\right]
$$

is $\alpha$-sectorial. Relations (3.5) can be proved in similar way as in [17]. We note that the form $v_{\widetilde{U}}$ is closed in the Hilbert space $\mathcal{D}\left[S_{K}\right]$.

Proposition 3.5 Suppose that the operator $W_{0}^{-1}$ is (+)-bounded in $\mathfrak{N}_{F}$. Then

1) the formula

$$
\widetilde{U}=\frac{1}{2} W_{0}^{-1}+\frac{1}{2} W_{0}^{-1 / 2} \widetilde{Z} W_{0}^{-1 / 2}
$$

gives one-to-one correspondence between $(+)$-m-accretive operators $\widetilde{U}$ in $\mathfrak{N}_{F}$, satisfying the condition (3.3) and (+)-contractions $\widetilde{Z}$ in $\overline{\mathfrak{N}_{0}}$,

2) the formula (3.8) gives one-to-one correspondence between operators $\widetilde{U}$ in $\mathfrak{N}_{F}$, satisfying the condition (3.4) and operators $\widetilde{Z}$ in $\overline{\mathfrak{N}_{0}}$, such that $\| \widetilde{Z} \sin \alpha \pm$ $i \cos \alpha I \|_{+} \leq 1\left(\Longleftrightarrow \widetilde{Z} \in C_{\overline{\mathfrak{N}_{0}}}(\alpha)\right)$.

Proof Boundness of $W_{0}^{-1}$ is equivalent to that the form $w_{0}$ defined by (2.26) is bounded from below in $\mathfrak{N}_{F}$, i.e. $w_{0}[e]=\left\|\widehat{S}_{F}^{-1 / 2} e\right\|_{+}^{2} \geq c\|e\|_{+}^{2}$ for all $e \in \mathfrak{N}_{0}$ with $c>0$. 
Because the condition (3.3) is equivalent to (3.6), we get for all $h \in \operatorname{Dom}(\widetilde{U})$ :

$$
\|\tilde{U} h\|_{+}\|h\|_{+} \geq \operatorname{Re}(\tilde{U} h, h)_{+} \geq w_{0}[\tilde{U} h] \geq c\|\widetilde{U} h\|_{+}^{2} .
$$

It follows that $\widetilde{U}$ is bounded in $\mathfrak{N}_{F}$ (with $\left.\operatorname{Ran}(\widetilde{U}) \subset \mathfrak{N}_{0}\right)$.

Let $W_{0}$ be the operator part of the relation $\mathbf{W}_{0}$. Then $W_{0}$ is $(+)$-nonnegative self-adjoint operator in $\overline{\mathfrak{N}}_{0}$, Ker $W_{0}^{-1}=\mathfrak{N}_{F} \ominus \mathfrak{N}_{0}$, and $W_{0}=\left(W_{0}^{-1} \uparrow \overline{\mathfrak{N}_{0}}\right)^{-1}$. Further we have for every $h \in \mathfrak{N}_{F}$ :

$$
\begin{aligned}
w_{0}[\tilde{U} h]-\operatorname{Re}(\tilde{U} h, h)_{+} & =\left\|W_{0}^{1 / 2} \widetilde{U} h\right\|_{+}^{2}-\operatorname{Re}(\tilde{U} h, h)_{+} \\
& =\left\|W_{0}^{1 / 2} \widetilde{U} h-\frac{1}{2} W_{0}^{-1 / 2} h\right\|_{+}^{2}-\frac{1}{4}\left\|W_{0}^{-1 / 2} h\right\|_{+}^{2} .
\end{aligned}
$$

Therefore, (3.6) is equivalent to

$$
\left\|W_{0}^{1 / 2} \widetilde{U} h-\frac{1}{2} W_{0}^{-1 / 2} h\right\|_{+}^{2} \leq \frac{1}{4}\left\|W_{0}^{-1 / 2} h\right\|_{+}^{2}, h \in \mathfrak{N}_{F} .
$$

This conditions is equivalent to the equality

$$
W_{0}^{1 / 2} \widetilde{U}-\frac{1}{2} W_{0}^{-1 / 2}=\frac{1}{2} \widetilde{Z} W_{0}^{-1 / 2}
$$

with some (+)-contraction $\widetilde{Z}$ in $\mathfrak{N}_{0}$. The last is equivalent to

$$
\widetilde{U}=\frac{1}{2} W_{0}^{-1}+\frac{1}{2} W_{0}^{-1 / 2} \widetilde{Z} W_{0}^{-1 / 2}
$$

One can verify that the condition

$$
\tan \alpha\left(\operatorname{Re}(\widetilde{U} h, h)_{+}-w_{0}[\tilde{U} h]\right) \geq\left|\operatorname{Im}(\widetilde{U} h, h)_{+}\right|
$$

for all $h \in \operatorname{Dom}(\widetilde{U})$ is equivalent to the following

$$
\left\|W_{0}^{1 / 2} \widetilde{U} h-\frac{1 \pm i \cot \alpha}{2} W_{0}^{-1 / 2} h\right\|_{+}^{2} \leq \frac{1}{4 \sin ^{2} \alpha}\left\|W_{0}^{-1 / 2} h\right\|_{+}^{2} .
$$

Because $\widetilde{U}$ satisfies (3.6), it has the representation (3.8) with (+)-contraction $\widetilde{Z}$. Hence (3.10) is equivalent

$$
\|(\widetilde{Z} \sin \alpha \pm i \cos \alpha I) e\|_{+}^{2} \leq\|e\|_{+}^{2}, e \in \overline{\mathfrak{N}}_{0}
$$


Observe that if the defect numbers of $S$ are finite, then the subspace $\mathfrak{N}_{F}$ is finitedimensional and because $w_{0}[e] \geq 2\|e\|^{2}$ for all $e \in \mathfrak{N}_{0}$, the form $w_{0}$ is $(+)$-positively definite in $\mathfrak{N}_{0}$. Therefore, the operator $W_{0}^{-1}$ is $(+)$-bounded.

In general case of unbounded operator $W_{0}^{-1}$ a description of all $\widetilde{U}$ satisfying (3.3) or (3.4) can be given by means of fractional-linear transformation of $\widetilde{U}$ and $\mathbf{W}_{0}$.

Let $\mathcal{W}_{0}$ be the linear fractional transformation of $\mathbf{W}_{0}$, i.e.

$$
\mathcal{W}_{0}\left(e+e^{\prime}\right)=e-e^{\prime}, \text { where }\left\langle e, e^{\prime}\right\rangle \in \mathbf{W}_{0}
$$

Then $\mathcal{W}_{0}$ is a $(+)$-contraction in $\mathfrak{N}_{F}$ and moreover

$$
\mathcal{W}_{0}=\left(W_{0}^{-1}-I\right)\left(W_{0}^{-1}+I\right)^{-1}
$$

Now from Theorems 3.4, 2.1 and Corollary 2.2 we obtain the following result

Theorem 3.6 There is the one-to-one correspondence between quasi-self-adjoint $m$-accretive extensions $\widetilde{S}$ of a nonnegative symmetric operator $S$ and $(+)$-contractions $\widetilde{\mathcal{Y}}$ in $\overline{\mathfrak{N}}_{0}$. This correspondence is given by the formulas

$$
\begin{aligned}
\operatorname{Dom}(\widetilde{S}) & =\operatorname{Dom}(S) \oplus\left(I+S_{F} \widetilde{U}\right) \operatorname{Dom}(\widetilde{U}) \\
\widetilde{U} & =(I-\widetilde{\mathcal{U}})(I+\widetilde{\mathcal{U}})^{-1} \\
\widetilde{\mathcal{U}} & =I-\frac{1}{2}\left(I+\mathcal{W}_{0}\right)^{1 / 2}(I+\widetilde{\mathcal{Y}})\left(I+\mathcal{W}_{0}\right)^{1 / 2}
\end{aligned}
$$

where $\mathcal{W}_{0}$ is given by (3.11). The extension $\widetilde{S}$ is quasi-self-adjoint and $m$ - $\alpha$-sectorial if and only if the operator $\widetilde{\mathcal{Y}}$ satisfies the condition

$$
\|\widetilde{\mathcal{Y}} \sin \alpha \pm i \cos \alpha I\|_{+} \leq 1\left(\Longleftrightarrow \mathcal{Y} \in C_{\overline{\mathfrak{N}}_{0}}(\alpha)\right)
$$

\subsection{Extremal m-Accretive Quasi-Self-Adjoint Extensions}

Definition 3.7 $[4,8]$ Quasi-self-adjoint m-accretive extension $\widetilde{S}$ of a nonnegative symmetric operator $S$ is called extremal if

$$
\inf \{\operatorname{Re}(\widetilde{S}(f-\psi), f-\psi), \psi \in \operatorname{Dom}(S)\}=0
$$

for all $f \in \operatorname{Dom}(\widetilde{S})$.

By means of fractional linear transformation $(I-\widetilde{S})(I+\widetilde{S})^{-1}$ the notion and characterization of quasi-self-adjoint extremal extensions were given in [14].

Proposition 3.8 The following conditions are equivalent:

(i) the quasi-self-adjoint m-accretive extension $\widetilde{S}$ of a nonnegative symmetric operator $S$ is extremal; 
(ii) the maximal (+)-accretive operator $\widetilde{U}$ in $\mathfrak{N}_{F}$ in the representation (3.2) satisfies the condition

$$
\operatorname{Ran}(\widetilde{U}) \subset \mathfrak{N}_{0} \text { and } \operatorname{Re}(\widetilde{U} h, h)_{+}=w_{0}[\widetilde{U} h], h \in \operatorname{Dom}(\widetilde{U})
$$

(iii) the maximal (+)-accretive operator $\widetilde{U}$ in $\mathfrak{N}_{F}$ in the representation (3.2) satisfies the condition

$$
\operatorname{Ran}(\widetilde{U}) \subset \mathfrak{N}_{0} \text { and } \operatorname{Re}\left(\left(\widetilde{U} P_{\widetilde{U}}\right)^{-1} e, e\right)_{+}=w_{0}[e] \text { for all } e \in \operatorname{Ran}(\widetilde{U}),
$$

where $P_{\widetilde{U}}$ is the $(+)$-orthogonal projection in $\mathfrak{N}_{F}$ onto $\overline{\operatorname{Ran}}(\widetilde{U})$;

(iv) the operator $\widetilde{\mathcal{Y}}$ in (3.12) is (+)-isometric in $\overline{\mathfrak{N}}_{0}$.

If the operator $W_{0}^{-1}$ is $(+)$-bounded then $\widetilde{S}$ given by (3.2) is extremal if and only if the operator $\widetilde{U}$ is of the form (3.8) with (+)-isometric operator $\widetilde{Z}$ in $\overline{\mathfrak{N}}_{0}$.

Proof Let $\widetilde{S}$ be a quasi-self-adjoint m-accretive extensions of $S$ and let $g \in \operatorname{Dom}(\widetilde{S})$. Then by (3.2) the vector $g$ has the representation $g=\varphi+h+S_{F} \widetilde{U} h$, where $h \in$ Dom $(\widetilde{U})$. We will use the relation (3.7):

$$
(\widetilde{S} g, g)-\left\|S_{K}^{1 / 2} g\right\|^{2}=(h, \widetilde{U} h)_{+}-w_{0}[\tilde{U} h] .
$$

Let $\psi \in \operatorname{Dom}(S)$. Then

$$
(\widetilde{S}(g-\psi), g-\psi)=\left\|S_{K}^{1 / 2}(g-\psi)\right\|^{2}+(h, \widetilde{U} h)_{+}-w_{0}[\tilde{U} h] .
$$

Now it follows from (2.30)

$$
\inf \{\operatorname{Re}(\widetilde{S}(g-\psi), g-\psi), \psi \in \operatorname{Dom}(S)\}=(h, \widetilde{U} h)_{+}-w_{0}[\widetilde{U} h]
$$

Therefore, the extension $\widetilde{S}$ is extremal if and only if $(h, \widetilde{U} h)_{+}=w_{0}[\widetilde{U} h]$ for every $h \in \operatorname{Dom}(\widetilde{U})$. Passing to the inverse in the last equality we get the equivalent condition (3.14).

In terms of the fractional linear transformations of $\widetilde{U}$ and $\mathbf{W}_{0}\left(W_{0}^{-1}\right)$ condition (3.13) takes the form (see (2.8))

$$
\operatorname{Re}((I-\tilde{\mathcal{U}}) e, e)_{+}-\left\|\left(I+\mathcal{W}_{0}\right)^{-1 / 2}(I-\tilde{\mathcal{U}}) e\right\|_{+}^{2}=0, e \in \mathfrak{N}_{F}
$$

Using the (3.12) we obtain

$$
\frac{1}{2} \operatorname{Re}((I+\tilde{\mathcal{Y}}) \varphi, \varphi)_{+}=\frac{1}{4}\|(I+\tilde{\mathcal{Y}}) \varphi\|_{+}^{2},
$$

where $\varphi=\left(I+\mathcal{W}_{0}\right)^{1 / 2} e$ which is the same as $\|\widetilde{\mathcal{Y}} \varphi\|_{+}=\|\varphi\|_{+}$for all $\varphi \in \overline{\mathfrak{N}}_{0}$. 
If the operator $W_{0}^{-1}$ is (+)-bounded then according (3.8) the condition (3.13) takes the form

$$
\frac{1}{2} \operatorname{Re}\left((I+\widetilde{Z}) W_{0}^{-1 / 2} e, W_{0}^{-1 / 2} e\right)_{+}=\frac{1}{4}\left\|(I+\widetilde{Z}) W_{0}^{-1 / 2} e\right\|_{+}^{2}, e \in \mathfrak{N}_{F},
$$

which is the same as $\|\widetilde{Z} \varphi\|_{+}=\|\varphi\|_{+}$for all $\varphi \in \overline{\mathfrak{N}}_{0}$.

\section{Symmetric Operator with Finite Defect Numbers}

Consider an operator $S$ with finite defect numbers.

Proposition 4.1 Suppose that nonnegative symmetric operator $S$ has defect numbers $\langle m, m\rangle, m \in \mathbb{N}, \mathfrak{N}_{0}=\mathfrak{N}_{F}$ and let $\left\{e_{1}, e_{2}, \ldots, e_{m}\right\}$ be a linear basis of the subspace $\mathfrak{N}_{F}$. Denote by $\mathcal{G}$ and $\mathcal{W}$ following $m \times m$ matrices:

$$
\mathcal{G}=\left\|\left(e_{k}, e_{j}\right)_{+}\right\|_{k, j=1}^{m}, \quad \mathcal{W}_{0}=\left\|w_{0}\left[e_{k}, e_{j}\right]\right\|_{k, j=1}^{m} .
$$

There is a one-to-one correspondence between

1) all $m$-accretive quasi-self-adjoint extensions of $S$ and all $m \times m$ matrices $\mathcal{U}=$ $\left\|u_{k j}\right\|_{k, j=1}^{m}$, satisfying the condition

$$
\mathcal{U G}+\mathcal{G U}^{*} \geq 2 \mathcal{U} \mathcal{W}_{0} \mathcal{U}^{*}
$$

2) all $m$ - $\alpha$-sectorial quasi-self-adjoint extensions of $S$ and all $m \times m$ matrices $\mathcal{U}=\left\|u_{k j}\right\|_{k, j=1}^{m}$, satisfying the condition

$$
\left\{\begin{array}{l}
\tan \alpha \cdot\left(\mathcal{U G}+\mathcal{G U}^{*}\right)+i\left(\mathcal{U G}-\mathcal{G U}^{*}\right) \geq 2 \tan \alpha \cdot \mathcal{U} \mathcal{W}_{0} \mathcal{U}^{*} \\
\tan \alpha \cdot\left(\mathcal{U G}+\mathcal{G U}^{*}\right)-i\left(\mathcal{U G}-\mathcal{G U}^{*}\right) \geq 2 \tan \alpha \cdot \mathcal{U} \mathcal{W}_{0} \mathcal{U}^{*}
\end{array}\right.
$$

This correspondence is given by the formulas

$$
\begin{gathered}
\operatorname{Dom}(\widetilde{S})=\left\{f+\sum_{j=1}^{m} \lambda_{j} e_{j}+\sum_{k, j=1}^{m} u_{k j} \lambda_{k} S_{F} e_{j}, f \in \operatorname{Dom}(S),\left(\lambda_{1}, \ldots, \lambda_{m}\right) \in \mathbb{C}^{m}\right\}, \\
\widetilde{S}\left(f+\sum_{j=1}^{m} \lambda_{j} e_{j}+\sum_{k, j=1}^{m} u_{k j} \lambda_{k} S_{F} e_{j}\right)=S_{F} f+\sum_{j=1}^{m} \lambda_{j} S_{F} e_{j}-\sum_{k, j=1}^{m} u_{k j} \lambda_{k} e_{j} .
\end{gathered}
$$

If $\mathcal{U}=\mathcal{G W}_{0}^{-1}$, then the corresponding extension is the Krĕn-von Neumann extension $S_{K}$. 
Proof Let $h=\sum_{j=1}^{m} \lambda_{j} e_{j} \in \mathfrak{N}_{F}$ and let $U$ be the operator in $\mathfrak{N}_{F}$ given by

$$
U\left(\sum_{j=1}^{m} \lambda_{j} e_{j}\right):=\sum_{k, j=1}^{m} u_{k j} \lambda_{k} e_{j}
$$

Then

$$
(U h, h)_{+}=\sum_{k, j=1}^{m} \lambda_{j} \bar{\lambda}_{k}\left(\sum_{l=1}^{m} u_{j l}\left(e_{l}, e_{k}\right)_{+}\right) .
$$

Observe that the matrix $\mathcal{W}=\left\|w_{k j}\right\|_{k, j=1}^{m}$ of the operator $W_{0}$ associated with the form $w_{0}[\cdot, \cdot]$ in the basis $\left\{e_{j}\right\}_{j=1}^{m}$ coincides with the matrix $\mathcal{W}_{0} \mathcal{G}^{-1}$. Indeed since

$$
w_{0}[h]=\left(W_{0} h, h\right)_{+}=\sum_{k, j=1}^{m} \lambda_{j} \bar{\lambda}_{k}\left(\sum_{l=1}^{m} w_{j l}\left(e_{l}, e_{k}\right)_{+}\right)
$$

and

$$
w_{0}[h]=\sum_{k, j=1}^{m} \lambda_{j} \bar{\lambda}_{k} w_{0}\left[e_{j}, e_{k}\right]
$$

we get $\mathcal{W}_{0}=\mathcal{W G}$.

Denote $g_{k j}=\left(e_{k}, e_{j}\right)_{+}$and $w_{k j}^{0}=w_{0}\left[e_{k}, e_{j}\right]$. Due to (4.3), (4.4) the condition

$$
\operatorname{Re}(U h, h)_{+} \geq \omega_{0}[U h], h \in \operatorname{Dom}(U)
$$

can be rewritten as follows

$$
\sum_{k, j=1}^{m} \lambda_{j} \bar{\lambda}_{k}\left(\sum_{s=1}^{m}\left(u_{j s} g_{s k}+g_{j s} \bar{u}_{k s}\right)-2 \sum_{s, l=1}^{m} u_{j s} w_{s l}^{0} \bar{u}_{k l}\right) \geq 0 .
$$

This yields

$$
\mathcal{U G}+\mathcal{G U}^{*}-2 \mathcal{U} \mathcal{W}_{0} \mathcal{U}^{*} \geq 0
$$

Extension $\widetilde{S}$ is $\mathrm{m}-\alpha$-sectorial iff the sesquilinear form

$$
q[h, e]:=(U h, e)_{+}-w_{0}[U h, U e]
$$

is $\alpha$-sectorial, i.e.

$$
|\operatorname{Im} q[h]| \leq \tan \alpha \cdot \operatorname{Re} q[h], h \in \operatorname{Dom}(U) .
$$


From (4.3) and (4.4) we get

$$
\begin{aligned}
& \operatorname{Re} q[h]=\frac{1}{2} \sum_{k, j=1}^{m} \lambda_{j} \overline{\lambda_{k}}\left(\sum_{s=1}^{m}\left(u_{j s} g_{s k}+g_{j s} \bar{u}_{k s}\right)-2 \sum_{s, l=1}^{m} u_{j s} w_{s l}^{0} \bar{u}_{k l}\right), \\
& \operatorname{Im} q[h]=\frac{1}{2 i} \sum_{k, j=1}^{m} \lambda_{j} \bar{\lambda}_{k}\left(\sum_{s=1}^{m}\left(u_{j s} g_{s k}-g_{j s} \bar{u}_{k s}\right)\right) .
\end{aligned}
$$

Then (4.6) becomes:

$$
\left\{\begin{array}{l}
\frac{1}{2 i} \sum_{k, j=1}^{m} \lambda_{j} \bar{\lambda}_{k}\left(\sum_{s=1}^{m}\left(u_{j s} g_{s k}-g_{j s} \bar{u}_{k s}\right)\right) \\
\leq \tan \alpha \cdot \frac{1}{2} \sum_{k, j=1}^{m} \lambda_{j} \overline{\lambda_{k}}\left(\sum_{s=1}^{m}\left(u_{j s} g_{s k}+g_{j s} \bar{u}_{k s}\right)-2 \sum_{s, l=1}^{m} u_{j s} w_{s l}^{0} \bar{u}_{k l}\right) \\
\frac{1}{2 i} \sum_{k, j=1}^{m} \lambda_{j} \bar{\lambda}_{k}\left(\sum_{s=1}^{m}\left(g_{j s} \bar{u}_{k s}-u_{j s} g_{s k}\right)\right) \\
\leq \tan \alpha \cdot \frac{1}{2} \sum_{k, j=1}^{m} \lambda_{j} \bar{\lambda}_{k}\left(\sum_{s=1}^{m}\left(u_{j s} g_{s k}+g_{j s} \bar{u}_{k s}\right)-2 \sum_{s, l=1}^{m} u_{j s} w_{s l}^{0} \bar{u}_{k l}\right)
\end{array}\right.
$$

The latter gives

$$
\left\{\begin{array}{l}
\frac{1}{2 i}\left(\mathcal{U G}-\mathcal{G U}^{*}\right) \leq \tan \alpha \cdot\left(\frac{1}{2}\left(\mathcal{U G}+\mathcal{G U}^{*}\right)-\mathcal{U W}_{0} \mathcal{U}^{*}\right) \\
\frac{1}{2 i}\left(\mathcal{G U}^{*}-\mathcal{U G}\right) \leq \tan \alpha \cdot\left(\frac{1}{2}\left(\mathcal{U G}+\mathcal{G U}^{*}\right)-\mathcal{U W}_{0} \mathcal{U}^{*}\right)
\end{array}\right.
$$

In the equivalent form

$$
\left\{\begin{array}{l}
\tan \alpha \cdot\left(\mathcal{U G}+\mathcal{G U}^{*}\right)+i\left(\mathcal{U G}-\mathcal{G U}^{*}\right) \geq 2 \tan \alpha \cdot \mathcal{U} \mathcal{W}_{0} \mathcal{U}^{*} \\
\tan \alpha \cdot\left(\mathcal{U G}+\mathcal{G U}^{*}\right)-i\left(\mathcal{U G}-\mathcal{G U}^{*}\right) \geq 2 \tan \alpha \cdot \mathcal{U} \mathcal{W}_{0} \mathcal{U}^{*}
\end{array}\right.
$$

The fact that $S_{K}$ is determined by $\mathcal{U}=\mathcal{G W}_{0}^{-1}$ is established in [17].

\section{5 m-Accretive Hamiltonians Corresponding to Finite Numbers of $\delta^{\prime}$ Interactions}

As application of our results we consider one example from solvable models of quantum mechanics [1]. Let $y_{1}, y_{2}, \ldots, y_{m} \in \mathbb{R}$. Consider linear operator:

$$
\left\{\begin{array}{l}
\operatorname{Dom}(S)=\left\{f \in W_{2}^{2}(\mathbb{R}): f^{\prime}\left(y_{j}\right)=0, j=1, \ldots, m\right\}, \\
S=-\frac{d^{2}}{d x^{2}}
\end{array}\right.
$$

where $W_{2}^{2}(\mathbb{R})$ is the Sobolev space. Operator $S$ densely defined symmetric and nonnegative operator in $L_{2}(\mathbb{R})$ with defect numbers $\langle m, m\rangle$. It can be proved that the 
Friedrichs extension $S_{F}$ of $S$ is given by:

$$
\operatorname{Dom}\left(S_{F}\right)=W_{2}^{2}(\mathbb{R}), \quad S_{F}=-\frac{d^{2}}{d x^{2}} .
$$

Using the Fourier transform:

$$
\hat{f}(p)=(\mathcal{F} f)(p)=\lim _{R \rightarrow \infty} \frac{1}{\sqrt{2 \pi}} \int_{-R}^{R} f(x) e^{-i p x} d x
$$

we obtain in the $p$-representation the nonnegative symmetric operator $A$ and its Friedrichs extension $A_{F}$ :

$$
\begin{aligned}
\operatorname{Dom}(A) & =\left\{h(p) \in L^{2}(\mathbb{R}, d p): \int_{\mathbb{R}} h(p) p \exp \left(i p y_{j}\right) d p=0, j=1, \ldots, m\right\}, \\
(A h)(p) & =p^{2} h(p), h(p) \in \operatorname{Dom}(A) \\
\operatorname{Dom}\left(A_{F}\right) & =H_{2}(\mathbb{R}):=L^{2}\left(\mathbb{R},\left(p^{4}+1\right) d p\right) \\
\left(A_{F} h\right)(p) & =p^{2} h(p), h(p) \in \operatorname{Dom}\left(A_{F}\right)
\end{aligned}
$$

Let $e_{j}(p)=p \frac{\exp \left(-i p y_{j}\right)}{1+p^{4}}, j=1, \ldots, m$, then

$$
\begin{aligned}
\mathfrak{N}_{F} & =\operatorname{span}\left\{e_{1}(p), \ldots, e_{m}(p)\right\} \\
\mathfrak{M}_{F} & =\operatorname{span}\left\{p^{2} e_{1}(p), \ldots, p^{2} e_{m}(p)\right\}
\end{aligned}
$$

The adjoint operator is given by

$$
\begin{aligned}
& \operatorname{Dom}\left(A^{*}\right)=\underset{\operatorname{Dom}}{ }(A) \dot{+} \mathfrak{N}_{F} \dot{+} \mathfrak{M}_{F}=H_{2}(\mathbb{R}) \dot{+} \mathfrak{M}_{F}, \\
& A^{*}\left(f(p)+\sum_{j=1}^{m} \lambda_{j} p^{2} e_{j}(p)\right)=p^{2} f(p)-\sum_{j=1}^{m} \lambda_{j} e_{j}(p), \\
& f(p) \in H_{2}(\mathbb{R}),\left(\lambda_{1}, \ldots, \lambda_{m}\right) \in \mathbb{C}^{m} .
\end{aligned}
$$

Since

$$
\begin{aligned}
\operatorname{Dom}\left(A_{F}^{1 / 2}\right) & =H_{1}(\mathbb{R}):=L^{2}\left(\mathbb{R},\left(p^{2}+1\right) d p\right), \\
\left(A_{F}^{1 / 2} f\right)(p) & =|p| f(p), f(p) \in H_{1}(\mathbb{R}),
\end{aligned}
$$

then

$$
A_{F}^{-1 / 2} e_{j}(p)=\frac{p \exp \left(-i p y_{j}\right)}{|p|\left(1+p^{4}\right)} \in H_{1}(\mathbb{R}), j=1, \ldots, m
$$


Clearly that $A_{F} \neq A_{K}$ and $\mathfrak{N}_{0}=\mathfrak{N}_{F}$. It follows that $\mathfrak{N}_{F}=\mathfrak{N}_{0}=\operatorname{Ran}\left(A_{F}^{1 / 2}\right) \cap \mathfrak{N}_{F}$. Hence, the Friedrichs and Kreı̆n extensions $A_{F}$ and $A_{K}$ are transversal. Providing direct calculation we obtain:

$$
\begin{aligned}
g_{k j} & =\left(e_{k}(p), e_{j}(p)\right)_{+}=\frac{\pi}{\sqrt{2}} \exp \left(-\frac{\left|y_{k}-y_{j}\right|}{\sqrt{2}}\right)\left(\cos \frac{\left|y_{k}-y_{j}\right|}{\sqrt{2}}-\sin \frac{\left|y_{k}-y_{j}\right|}{\sqrt{2}}\right), \\
\omega_{k j} & =\left(A_{F}^{1 / 2} e_{k}(p), A_{F}^{1 / 2} e_{j}(p)\right)+\left(A_{F}^{-1 / 2} e_{k}(p), A_{F}^{-1 / 2} e_{j}(p)\right) \\
& =\frac{\pi}{\sqrt{2}} \exp \left(-\frac{\left|y_{k}-y_{j}\right|}{\sqrt{2}}\right)\left(\cos \frac{\left|y_{k}-y_{j}\right|}{\sqrt{2}}+\sin \frac{\left|y_{k}-y_{j}\right|}{\sqrt{2}}\right) .
\end{aligned}
$$

Let

$$
\mathcal{W}_{0}=\left\|\omega_{k j}\right\|_{k, j=1}^{m}, \mathcal{G}=\left\|g_{k j}\right\|_{k, j=1}^{m}
$$

From Proposition 4.1 we obtain next description of 1) all $m$-accretive quasi-selfadjoint extensions $\widetilde{A}$ of $A, 2$ ) all $\mathrm{m}-\alpha$-sectorial quasi-self-adjoint extensions $\widetilde{A}$ of $A$ :

$$
\begin{aligned}
& \operatorname{Dom}(\widetilde{A})=\left\{f_{0}(p)+\sum_{j=1}^{m} \lambda_{j} e_{j}(p)+\sum_{k, j=1}^{m} u_{k j} \lambda_{k} p^{2} e_{j}(p)\right\}, \\
& f_{0}(p) \in \operatorname{Dom}(A),\left(\lambda_{1}, \ldots, \lambda_{m}\right) \in \mathbb{C}^{m}, \\
& \widetilde{A}\left(f_{0}(p)+\sum_{j=1}^{m} \lambda_{j} e_{j}(p)+\sum_{k, j=1}^{m} u_{k j} \lambda_{k} p^{2} e_{j}(p)\right) \\
& =p^{2} f_{0}(p)+\sum_{j=1}^{m} \lambda_{j} p^{2} e_{j}(p)-\sum_{k, j=1}^{m} u_{k j} \lambda_{k} e_{j}(p),
\end{aligned}
$$

where the matrices $\mathcal{U}=\left\|u_{k j}\right\|_{k, j=1}^{m}$ satisfies the condition:

$$
\begin{aligned}
& \text { 1) } \mathcal{U G}+\mathcal{G U}^{*} \geq 2 \mathcal{U} \mathcal{W}_{0} \mathcal{U}^{*} \\
& \text { 2) }\left\{\begin{array}{l}
\tan \alpha \cdot\left(\mathcal{U G}+\mathcal{G} \mathcal{U}^{*}\right)+i\left(\mathcal{U G}-\mathcal{G U}^{*}\right) \geq 2 \tan \alpha \cdot \mathcal{U} \mathcal{W}_{0} \mathcal{U}^{*} \\
\tan \alpha \cdot\left(\mathcal{U G}+\mathcal{G} \mathcal{U}^{*}\right)-i\left(\mathcal{U G}-\mathcal{G U}^{*}\right) \geq 2 \tan \alpha \cdot \mathcal{U} \mathcal{W}_{0} \mathcal{U}^{*}
\end{array}\right.
\end{aligned}
$$

In case, when $m=1$ we get:

$$
\begin{aligned}
& \operatorname{Dom}(\widetilde{A})=\left\{f_{0}(p)+\lambda \frac{\left(1+u p^{2}\right) \exp (-i p y)}{1+p^{4}}\right\} \\
& \widetilde{A}\left(f_{0}(p)+\lambda \frac{\left(1+u p^{2}\right) \exp (-i p y)}{1+p^{4}}\right)=p^{2} f_{0}(p)+\lambda p \frac{\left(p^{2}-u\right) \exp (-i p y)}{1+p^{4}}, \\
& f_{0}(p) \in \operatorname{Dom}(A), \lambda \in \mathbb{C}, y \in \mathbb{R}, \\
& \left(\operatorname{Re} u-\frac{1}{2}\right)^{2}+(\operatorname{Im} u)^{2} \leq \frac{1}{4} \text { for m-accretive extensions, }
\end{aligned}
$$


$\left(\operatorname{Re} u-\frac{1}{2}\right)^{2}+\left(\operatorname{Im} u \pm \frac{\cot \alpha}{2}\right)^{2} \leq \frac{1}{4 \sin ^{2} \alpha}$ for $\mathrm{m}$ - $\alpha$-sectorial extensions.

The inverse Fourier transform $\mathcal{F}^{-1}$ is given by the next equality

$$
\mathcal{F}^{-1} \hat{f}=f(x)=\lim _{R \rightarrow \infty} \frac{1}{\sqrt{2 \pi}} \int_{-R}^{R} \hat{f}(p) \exp (i p x) d p .
$$

We have $S=\mathcal{F}^{-1} A \mathcal{F}, S_{F}=\mathcal{F}^{-1} A_{F} \mathcal{F}$.

Providing direct calculation we obtain:

$$
\begin{aligned}
\mathcal{F}^{-1} e_{j}(p) & =g_{j}(x)=i \sqrt{\frac{\pi}{2}} \exp \left(-\frac{\left|x-y_{j}\right|}{\sqrt{2}}\right) \sin \left(\frac{\left|x-y_{j}\right|}{\sqrt{2}}\right), \\
\mathcal{F}^{-1} A_{F} e_{j}(p) & =h_{j}(x)=i \sqrt{\frac{\pi}{2}} \exp \left(-\frac{\left|x-y_{j}\right|}{\sqrt{2}}\right) \cos \left(\frac{\left|x-y_{j}\right|}{\sqrt{2}}\right) .
\end{aligned}
$$

Since $\mathcal{F}$ the unitary operator we obtain next theorem.

Theorem 5.1 Let operator $S$ is defined as (5.1). Then formulas

$$
\begin{aligned}
& \operatorname{Dom}(\widetilde{S})=\left\{f_{0}(x)+\sum_{j=1}^{m} \lambda_{j} g_{j}(x)+\sum_{k, j=1}^{m} u_{k j} \lambda_{k} h_{j}(x)\right\}, \\
& f_{0}(x) \in \operatorname{Dom}(S),\left(\lambda_{1}, \ldots, \lambda_{m}\right) \in \mathbb{C}^{m}, \\
& \widetilde{S}\left(f_{0}(x)+\sum_{j=1}^{m} \lambda_{j} g_{j}(x)+\sum_{k, j=1}^{m} u_{k j} \lambda_{k} h_{j}(x)\right) \\
& =-\frac{d^{2}}{d x^{2}} f_{0}(x)+\sum_{j=1}^{m} \lambda_{j} h_{j}(x)-\sum_{k, j=1}^{m} u_{k j} \lambda_{k} g_{j}(x),
\end{aligned}
$$

give one-to-one correspondence between of

1) all m-accretive quasi-self-adjoint extensions $\widetilde{S}$ of $S$ and all matrices $\mathcal{U}=$ $\left\|u_{k j}\right\|_{k, j=1}^{m}$ satisfying the condition

$$
\mathcal{U G}+\mathcal{G U}^{*} \geq 2 \mathcal{U} \mathcal{W}_{0} \mathcal{U}^{*}
$$

2) all $m$ - $\alpha$-sectorial quasi-self-adjoint extensions $\widetilde{S}$ of $S$ and all matrices $\mathcal{U}=$ $\left\|u_{k j}\right\|_{k, j=1}^{m}$ satisfying the condition

$$
\left\{\begin{array}{l}
\tan \alpha \cdot\left(\mathcal{U G}+\mathcal{G U}^{*}\right)+i\left(\mathcal{U G}-\mathcal{G U}^{*}\right) \geq 2 \tan \alpha \cdot \mathcal{U} \mathcal{W}_{0} \mathcal{U}^{*} \\
\tan \alpha \cdot\left(\mathcal{U G}+\mathcal{G U}^{*}\right)-i\left(\mathcal{U G}-\mathcal{G U}^{*}\right) \geq 2 \tan \alpha \cdot \mathcal{U} \mathcal{W}_{0} \mathcal{U}^{*}
\end{array}\right.
$$


In particular, if $m=1$ then

$$
\begin{aligned}
& \operatorname{Dom}(\widetilde{S})=\left\{f_{0}(x)+\lambda \exp \left(-\frac{|x-y|}{\sqrt{2}}\right)\left(\sin \frac{|x-y|}{\sqrt{2}}+u \cos \frac{|x-y|}{\sqrt{2}}\right)\right\}, \\
& f_{0}(x) \in \operatorname{Dom}(S), \quad \lambda \in \mathbb{C}, \quad y \in \mathbb{R}, \\
& \left(\operatorname{Re} u-\frac{1}{2}\right)^{2}+(\operatorname{Im} u)^{2} \leq \frac{1}{4} \text { for m-accretive extensions, } \\
& \left(\operatorname{Re} u-\frac{1}{2}\right)^{2}+\left(\operatorname{Im} u \pm \frac{\cot \alpha}{2}\right)^{2} \leq \frac{1}{4 \sin ^{2} \alpha} \text { for } m \text { - } \alpha \text {-sectorial extensions } \\
& \widetilde{S}\left(f_{0}(x)+\lambda \exp \left(-\frac{|x-y|}{\sqrt{2}}\right)\left(\sin \frac{|x-y|}{\sqrt{2}}+u \cos \frac{|x-y|}{\sqrt{2}}\right)\right) \\
& =-\frac{d^{2}}{d x^{2}} f_{0}(x)+\lambda \exp \left(-\frac{|x-y|}{\sqrt{2}}\right)\left(\cos \frac{|x-y|}{\sqrt{2}}-u \sin \frac{|x-y|}{\sqrt{2}}\right) .
\end{aligned}
$$

\section{Resolvents of Quasi-Self-Adjoint m-Accretive Extensions}

\subsection{Boundary Triplets and Abstract Boundary Conditions}

Recall the definition of the boundary triplet (boundary value space) [33,34].

Definition 6.1 The triplet $\left\{\mathcal{H}, \Gamma_{1}, \Gamma_{0}\right\}$ is called a boundary triplet of $S^{*}$ if $\mathcal{H}$ is a Hilbert space and $\Gamma_{0}, \Gamma_{1}$ are bounded linear operators from the Hilbert space $H_{+}=\operatorname{Dom}\left(S^{*}\right)$ with the graph norm into $\mathcal{H}$ such that the map $\vec{\Gamma}=\left\langle\Gamma_{0}, \Gamma_{1}\right\rangle$ is a surjection from $H_{+}$ onto $\mathcal{H}^{2}$ and the Green identity holds:

$$
\left(S^{*} f, g\right)-\left(f, S^{*} g\right)=\left(\Gamma_{1} f, \Gamma_{0} g\right)_{\mathcal{H}}-\left(\Gamma_{0} f, \Gamma_{1} g\right)_{\mathcal{H}} \quad \text { for all } f, g \in H_{+}
$$

The relations

$$
\operatorname{Dom}(\widetilde{S})=\left\{u \in \operatorname{Dom}\left(S^{*}\right): \vec{\Gamma} u \in \widetilde{\mathbf{T}}\right\}, \widetilde{S}=S^{*} \uparrow \operatorname{Dom}(\widetilde{S})
$$

give a one-to-one correspondence between all proper extensions $\widetilde{S}$ of $S\left(S \subset \widetilde{S} \subset S^{*}\right.$ ) and all linear relations $\widetilde{\mathbf{T}}$ in $\mathcal{H}$. An extension $\widetilde{S}$ is a self-adjoint one if and only if the relation $\widetilde{\mathbf{T}}$ is self-adjoint in $\mathcal{H}$.

As it was shown in $[24,25]$ the operators $S_{0}, S_{1}$ defined as follows

$$
S_{k}=S^{*} \uparrow \operatorname{Ker} \Gamma_{k}, k=0,1
$$

are transversal to each other self-adjoint extensions of $S$. The function $\Gamma_{0}(\lambda)=$ $\left(\Gamma_{0}\left\lceil\mathfrak{N}_{\lambda}\right)^{-1}\right.$ is the $\gamma$-field corresponding to $S_{0}[39,40]$. Note that as a consequence of (6.1) one can obtain the equality

$$
\Gamma_{0}(\bar{\lambda})=\left(\Gamma_{1}\left(S_{0}-\lambda I\right)^{-1}\right)^{*}
$$


Derkach and Malamud [23-25] define the Weyl (Weyl-Titchmarsh) function $M_{0}(\lambda)$ by the equality

$$
M_{0}(\lambda)=\Gamma_{1} \Gamma_{0}(\lambda) .
$$

The function $M_{0}$ is KreǏn-Langer $Q$-function [39,40]. In terms of boundary triplet the connection between a self-adjoint extension $\widetilde{S}_{\widetilde{T}}$ defined by relations (6.2) and its resolvent is given by

$$
\left(\widetilde{S}_{\widetilde{\mathbf{T}}}-\lambda I\right)^{-1}=\left(S_{0}-\lambda I\right)^{-1}+\Gamma_{0}(\lambda)\left(\widetilde{\mathbf{T}}-M_{0}(\lambda)\right)^{-1} \Gamma_{0}^{*}(\bar{\lambda}) .
$$

The triplet $\left\{\mathcal{H},-\Gamma_{0}, \Gamma_{1}\right\}$ also forms a boundary triplet of $S$ and the $\gamma$-field $\Gamma_{1}(\lambda)=$ $\left(\Gamma_{1} \uparrow \mathfrak{N}_{\lambda}\right)^{-1}$ corresponding to the self-adjoint extension $S_{1}$ determines the WeylTitchmarsh function $M_{1}(\lambda)=-\Gamma_{0} \Gamma_{1}(\lambda)$ which is connected with $M_{0}(\lambda)$ by the relation $M_{1}(\lambda)=-M_{0}^{-1}(\lambda)$.

Let $S$ be a nonnegative symmetric operator and let $S_{0}=S_{0}^{*} \geq 0$ be an extension of $S$. Choose the boundary triplet $\left\{\mathcal{H}, \Gamma_{1}, \Gamma_{0}\right\}$ such that $\operatorname{Ker} \Gamma_{0}=\operatorname{Dom}\left(S_{0}\right)$. It was established [23-25] (see also [26,31,32,42]) the following theorem.

Theorem 6.2 Let $S$ be a closed nonnegative symmetric operator. Then $S$ has a nonunique nonnegative self-adjoint extension if and only if

$$
\mathcal{D}=\left\{h \in \mathcal{H}: \lim _{x \uparrow 0}\left(M_{0}(x) h, h\right)_{\mathcal{H}}<\infty\right\} \neq\{0\},
$$

and the quadratic form

$$
\tau[h]=\lim _{x \uparrow 0}\left(M_{0}(x) h, h\right)_{\mathcal{H}}, \mathcal{D}[\tau]=\mathcal{D}
$$

is bounded from below. If $M_{0}(0)$ is a self-adjoint linear relation in $\mathcal{H}$ associated with $\tau$, then the Kre $\breve{n}-v o n$ Neumann extension $S_{K}$ can be defined by the boundary condition

$$
\operatorname{Dom}\left(S_{K}\right)=\left\{u \in \operatorname{Dom}\left(S^{*}\right):\left\langle\Gamma_{0} u, \Gamma_{1} u\right\rangle \in M_{0}(0)\right\}
$$

The relation $M_{0}(0)$ is also the strong resolvent limit of $M_{0}(x)$ when $x \rightarrow-0$. Moreover, $S_{0}$ and $S_{K}$ are disjoint iff $\overline{\mathcal{D}}=\mathcal{H}$ and transversal iff $\mathcal{D}=\mathcal{H}$. In addition, if $S_{0}=S_{F}$, then there is a one-to-one correspondence given by (6.2) between nonnegative self-adjoint extensions $\widetilde{S}_{\widetilde{\mathbf{T}}}$ and self-adjoint relations $\widetilde{\mathbf{T}}$ satisfying the condition

$$
\widetilde{\mathbf{T}} \geq M_{0}(0)
$$


6.2 Special Boundary Triplet and Description of Resolvents of Quasi-Self-Adjoint m-Accretive Extensions

Denote by $P_{\mathfrak{M}_{F}}^{+}$the orthogonal projection in $H_{+}$onto $\mathfrak{M}_{F}$. Put

$$
\mathcal{H}=\mathfrak{N}_{F}, \Gamma_{0}=-S^{*} P_{\mathfrak{M}_{F}}^{+}, \Gamma_{1}=P_{\mathfrak{N}_{F}}^{+}
$$

Using the relations $S^{*} S_{F} e=-e, e \in \mathfrak{N}_{F}$ and $S_{F} S^{*} h=-h, h \in \mathfrak{M}_{F}=S_{F} \mathfrak{N}_{F}$ one can easily check that the triplet $\left\{\mathfrak{N}_{F}, \Gamma_{1}, \Gamma_{0}\right\}$ is a boundary triplet for $S^{*}, \operatorname{Ker} \Gamma_{0}=$ $\operatorname{Dom}\left(S_{F}\right)$ and

$$
\Gamma_{0}(\lambda) e=\left(S_{F}-\lambda I\right)^{-1}\left(I+\lambda S_{F}\right) e+S_{F} e, e \in \mathfrak{N}_{F}
$$

is the $\gamma$-field corresponding to $S_{F}$. The Weyl-Titchmarsh function (6.4) in this case takes the form

$$
M_{0}(\lambda)=P_{\mathfrak{N}_{F}}^{+}\left(S_{F}-\lambda I\right)^{-1}\left(I+\lambda S_{F}\right) \uparrow \mathfrak{N}_{F}
$$

It is easy to verify that from (6.3) follows the relation

$$
\Gamma_{0}^{*}(\bar{\lambda})=P_{\mathfrak{N}_{F}}^{+}\left(S_{F}-\lambda I\right)^{-1}
$$

The next statement is established in [17].

Proposition 6.3 Suppose that $\operatorname{Ran}\left(S_{F}^{1 / 2}\right) \cap \mathfrak{N}_{F}=\mathfrak{N}_{0} \neq\{0\}$. Then

$$
\begin{aligned}
& \mathfrak{N}_{0}=\left\{e \in \mathfrak{N}_{F}: \lim _{x \uparrow 0}\left(M_{0}(x) e, e\right)_{+}<\infty\right\}, \\
& \lim _{x \uparrow 0}\left(M_{0}(x) e, e\right)_{+}=w_{0}[e], e \in \mathfrak{N}_{0} .
\end{aligned}
$$

Now we obtain that the linear relation $\mathbf{W}_{0}$ is associated with the closed quadratic form

$$
\lim _{x \uparrow 0}\left(M_{0}(x) e, e\right)_{+}=w_{0}[e], e \in \mathfrak{N}_{0}
$$

Let $\widetilde{S}$ be a quasi-self-adjoint m-accretive extension of $S$. By Theorem 2.8 we have

$$
\operatorname{Dom}(\widetilde{S})=\operatorname{Dom}(S) \dot{+}\left(I+S_{F} \widetilde{U}\right) \operatorname{Dom}(\widetilde{U}),
$$

where the (+)-m-accretive operator $\widetilde{U}$ satisfies condition (3.3). From (6.7) for $\vec{\Gamma}=$ $\left\langle\Gamma_{0}, \Gamma_{1}\right\rangle$ we get

$$
\vec{\Gamma} \operatorname{Dom}(\widetilde{S})=\{\langle\widetilde{U} e, e\rangle, e \in \operatorname{Dom}(\widetilde{U})\}
$$


or

$\operatorname{Dom}(\widetilde{S})=\left\{u \in \operatorname{Dom}\left(S^{*}\right): \Gamma_{0} u=\widetilde{U} \Gamma_{1} u\right\}, \operatorname{Re}(\widetilde{U} e, e)_{+} \geq w_{0}[\widetilde{U} e], e \in \operatorname{Dom}(\widetilde{U})$

So, we obtain the description of all quasi-self-adjoint m-accretive extensions in terms of boundary conditions. Now using (6.5) we get the following theorem.

Theorem 6.4 The formula

$$
\begin{aligned}
(\widetilde{S}-\lambda I)^{-1}= & \left(S_{F}-\lambda I\right)^{-1} \\
& +\left[\left(S_{F}-\lambda I\right)^{-1}\left(I+\lambda S_{F}\right)+S_{F}\right] \widetilde{U}\left(I-M_{0}(\lambda) \widetilde{U}\right)^{-1} P_{\mathfrak{N}_{F}}^{+}\left(S_{F}-\lambda I\right)^{-1}
\end{aligned}
$$

establishes a one-to-one correspondence between all quasi-self-adjoint m-accretive extensions of $S$ and all m-accretive operators $\widetilde{U}$ in $\mathfrak{N}_{F}$ satisfying condition (3.3).

Open Access This article is distributed under the terms of the Creative Commons Attribution Noncommercial License which permits any noncommercial use, distribution, and reproduction in any medium, provided the original author(s) and source are credited.

\section{References}

1. Albeverio, S., Gesztesy, F., Hoegh-Krohn, R., Holden, H.: Solvable models in quantum mechanics. In: Texts and Monographs in Physics. Springer, Berlin-New York (1988)

2. Ando, T., Nishio, K.: Positive self-adjoint extensions of positive symmetric operators. Tohóku Math. J. 22, 65-75 (1970)

3. Arlinskiŭ, Yu.M.: A class of contractions in a Hilbert space. Ukrain. Math. J. 39(6), 691-696 (1987, in Russian). English translation in Ukr. Math. J. 39(6), 560-564 (1987)

4. Arlinskiŭ, Yu.M., Positive spaces of boundary values and sectorial extensions of nonnegative symmetric operators. Ukrain. Math. Zh. 40(1), 8-14 (1988, in Russian). English translation in Ukr. Math. J. 40(1), 5-10 (1988)

5. Arlinskiı̌, Yu.M.: Characteristic functions of operators of the class $C(\alpha)$. Izv. Vyssh. Uchebn. Zaved. Mat. N.2, 13-21 (1991)

6. Arlinskiı̌, Yu.M.: On proper accretive extensions of positive linear relations. Ukr. Math. J. 47(6), 723730 (1995)

7. Arlinskiı̌, Yu.: Maximal sectorial extensions and closed forms associated with them. Ukr. Math. J. 48(6), 723-739 (1996, in Russian). English translation in Ukr. Math. J. 48(6), 809-827 (1996)

8. Arlinskiı̆, Yu.: Extremal extensions of sectorial linear relations. Matematychnii Studii 7(1), 81-96 (1997)

9. Arlinskiŭ, Y., Hassi, S., Sebestyen, Z., de Snoo, H.: On the class of extremal extensions of a nonnegative operators. Oper. Theory: Adv. Appl. 127, 41-81 (2001)

10. Arlinskiŭ, Yu., Kovalev, Y., Tsekanovskiŭ, E.: Quasi-self-adjoint maximal accretive extensions of nonnegative symmetric operators. TEKA Kom. Motor. i Energ. Roln.-OL PAN, XA, 6-14 (2010)

11. Arlinskiŭ, Yu.M., Tsekanovskiŭ, E.R.: Non-self-adjoint contractive extensions of Hermitian contractions and M.G. Krel̆n's theorems. Uspekhi Mat. Nauk 37(1), 131-132 (1982)

12. Arlinskiŭ, Yu.M., Tsekanovskiı̌, E.R.: Generalized resolvents of quasi-self-adjoint contracting extensions of a Hermitian contraction. Ukr. Mat. Zh. 35(5) (1983)

13. Arlinskiŭ, Yu., Tsekanovskiı̆, E.: On sectorial extensions of positive hermitian operators and their resolvents. Dokl. Akad. Nauk Armenian SSR 5, 199-202 (1984)

14. Arlinskiŭ, Yu.M., Tsekanovskiŭ, E.R.: Quasi-self-adjoint contractive extensions of Hermitian contractions. Teor. Funkts., Funkts. Anal. Prilozhen 50, 9-16 (1988, in Russian). English translation in J. Math. Sci. 49(6), 1241-1247 (1990) 
15. Arlinskiŭ, Yu., Tsekanovskiŭ, E.: On the theory of non-negative self-adjoint extensions of a non-negative symmetric operator. Report of National Academy of Scinces of Ukraine, vol. 11, pp. 30-37 (2002)

16. Arlinskiŭ, Yu., Tsekanovskiı̆, E.: On von Neumann's problem in extension theory of nonnegative operators. Proc. AMS 131(10), 3143-3154 (2003)

17. Arlinskiı̆, Yu., Tsekanovskǐ̆, E.: The von Neumann problem for nonnegative symmetric operators. Integr. Equ. Oper. Theory 51, 319-356 (2005)

18. Arlinskiŭ, Yu., Tsekanovskiı̆, E.: Krein's research on semi-bounded operators, its contemporary developments, and applications. Oper. Theory: Adv. Appl. 190, 65-112 (2009)

19. Arsene, Gr., Geondea, A.: Completing matrix contractions. J. Oper. Theory 7, 179-189 (1982)

20. Coddington, E.A., de Snoo, H.S.V.: Positive self-adjoint extensions of positive symmetric subspaces. Math. Z. 159, 203-214 (1978)

21. Crandall, M.G.: Norm preserving extensions of linear transformations in Hilbert space. Proc. Am. Math. Soc. 21, 335-340 (1969)

22. Davis, Ch., Kahan, W.M., Weinberger, H.F.: Norm preserving dilations and their applications to optimal error bounds. SIAM J. Numer. Anal. 19(3), 445-469 (1982)

23. Derkach, V.A., Malamud, M.M.: Weyl function of Hermitian operator andits connection with the characteristic function. Preprint 85-9, Fiz.-Tekhn. Inst. Akad. Nauk Ukraine, p. 50 (1985, in Russian)

24. Derkach, V.A., Malamud, M.M.: Generalized resolvents and the boundary value problems for Hermitian operators with gaps. J. Funct. Anal. 95(1), 1-95 (1991)

25. Derkach, V.A., Malamud, M.M.: The extension theory of Hermitian operators and the moment problem. J. Math. Sci. 73(2), 141-242 (1995)

26. Derkach, V.A., Malamud, M.M., Tsekanovskiı̌, E.R.: Sectorial extensions of positive operators and characteristic functions. Ukr. Math. J. 41(2), 151-158 (1989, in Russian). English tranlation in Ukr. Math. J. 41(2), 136-142 (1989)

27. Douglas, R.G.: On majorization, factorization and range inclusion of operators in Hilbert space. Proc. Am. Math. Soc. 17, 413-416 (1966)

28. Evans, W.D., Knowles, I.I.: On the extensions problem for accretive differential operators. J. Funct. Anal. 63(3), 276-298 (1985)

29. Evans, W.D., Knowles, I.: On the extension problem for singular accretive differential operators. J. Differ. Equ. 63, 264-288 (1986)

30. Fillmore, P.A., Williams, J.P.: On operator ranges. Adv. Math. 7, 254-281 (1971)

31. Gesztesy, F., Kalton, N., Makarov, K., Tsekanovskiŭ, E.: Some aplications of operator-valued Herglotz functions. Oper. Theory, Adv. Appl. 123, 271-321 (2001)

32. Gesztesy, F., Tsekanovskiŭ, E.: On matrix-valued Herglotz functions. Math. Nachr. 218, 61-138 (2000)

33. Gorbachuk, M.L., Gorbachuk, V.I.: Boundary value problems for differential-operator equations. Naukova Dumka Kiev (1984, in Russian)

34. Gorbachuk, V.I., Gorbachuk, M.L., Kochuber̆, A.N.: Extension theory of symmetric operators and boundary value problems. Ukr. Mat. Z. 41(10), 1298-1313 (1989)

35. Kato, T.: Perturbation Theory for Linear Operators. Springer, Berlin, Heidelberg, pp. 619 (1995)

36. Kochubei, A.N.: Extensions of a positive definite symmetric operator. Dokl. Akad. Nauk Ukrain. SSR, Ser. A, 3, 168-171 (1979, in Russian)

37. Kreı̆n, M.G.: The theory of self-adjoint extensions of semibounded Hermitian transformations and its applications, I. Mat. Sbornik 20(3), 431-495 (1947, in Russian)

38. Kreı̆n, M.G.: The theory of self-adjoint extensions of semibounded Hermitian transformations and its applications, II. Mat. Sbornik 21(3), 365-404 (1947, in Russian)

39. Kreı̆n, M.G., Langer, H.: Über die Q-function eines $\Pi$-Hermiteschen operators im raum $\Pi_{\kappa}$. Acta Sci. Math. Szeged 34, 191-230 (1973)

40. Krĕ̌n, M.G., Langer, H.: On defect subspaces and generalized resolvents of Hermitian operator in the space $\Pi_{\kappa}$. Fuct. Anal. Appl. 5(3), 54-69 (1971, in Russian)

41. Kreı̆n, M.G., Ovčarenko, I.E.: On $Q$-functions and sc-resolvents of nondensely defined Hermitian contractions. Siberian Math. J. 18, 728-746 (1977, in Russian)

42. Malamud, M.M.: On some classes of Hermitian operators with gaps. Ukr. Mat. J. 44(2), 215-234 (1992, in Russian)

43. Mikhailets, V.A.: Solvable and sectorial boundary value problems for the operator Sturm-Liouville equation. Ukr. Math. Zh. 26, 450-459 (1974, in Russian)

44. Mil'yo, O.Ya., Storozh, O.G.: On the general form of a maximally accretive extension of a positivedefinite operator. Dokl Akad. Nauk Ukraine 6, 19-22 (1991, in Russian) 
45. Phillips, R.: Dissipative operators and hyperbolic systems of partial differential equations. Trans. Am. Math. Soc. 90, 192-254 (1959)

46. Phillips, R.: On dissipative operators. In: Lectures in Differential Equations, vol. II, pp. 65-113. Van Nostrand-Reinhold, New York (1965)

47. Rofe-Beketov, F.S.: Numerical range of a linear relation and maximal relations. Theory of functions. Funct. Anal. Appl. 44, 103-112 (1985, in Russian)

48. Sz.-Nagy, B., Foias, C.: Harmonic Analysis of Operators on Hilbert Space. North-Holland, New York (1970)

49. Shmul'yan, Yu.L., Yanovskaya, R.N.: Blocks of a contractive operator matrix. Izv. Vyssh. Uchebn. Zaved. Mat. 7, 72-75 (1981)

50. Tsekanovskiı̆, E.: Non-self-adjoint accretive extensions of positive operators and theorems of Friedrichs-Krel̆n-Phillips. Funk. Anal. i Prilozhen. 14(2), 87-89 (1980, in Russian) 